TITLE:

\title{
Cognitive and neural underpinnings of goal maintenance in young children
}

$\operatorname{AUTHOR}(S):$

Yanaoka, Kaichi/ Moriguchi, Yusuke/ Saito, Satoru

\section{CITATION:}

Yanaoka, Kaichi/ Moriguchi, Yusuke/ Saito, Satoru. Cognitive and neural underpinnings of goal maintenance in young children. Cognition 2020, 203: 104378.

\section{ISSUE DATE:}

2020

URL:

http://hdl.handle.net/2433/259206

\section{RIGHT:}

(c) 2020. This manuscript version is made available under the CC-BY-NC-ND 4.0 license

http://creativecommons.org/licenses/by-nc-nd/4.0/.; The full-text file will be made open to the public on 1 October 2021 in accordance with publisher's 'Terms and Conditions for Self-Archiving'., この論文は出版社版でありません。引 用の際には出版社版をご確認ご利用ください。; This is not the published version. Please cite only the published version. 
This paper is not the copy of record and may not exactly replicate the final, authoritative version of the article. The final article is available via its DOI shown below.

Yanaoka, K., Moriguchi, Y., \& Saito, S. (2020). Cognitive and neural underpinnings of goal maintenance in young children. Cognition, 203, 104378.

https://doi.org/10.1016/j.cognition.2020.104378 


\begin{abstract}
Active maintenance of goal representations is an integral part of our mental regulatory processes. Previous developmental studies have highlighted goal neglect, which is the phenomenon caused by a failure to maintain goal representations, and demonstrated developmental changes of the ability to maintain goal representations among preschoolers. Yet, few studies have explored the cognitive mechanisms underlying preschoolers' development of goal maintenance. The first aim of this study was to test whether working memory capacity and inhibitory control contribute to goal maintenance using a paradigm for measuring goal neglect. Moreover, although recent studies have shown that preschoolers recruit lateral prefrontal regions in performing executive functions tasks, they could not specify the neural underpinnings of goal maintenance. Thus, the second aim was to examine whether lateral prefrontal regions played a key role in maintaining goal representations using functional near-infrared spectroscopy. Our results showed that developmental differences in inhibitory control predicted the degree of goal neglect. It was also demonstrated that activation in the right prefrontal region was associated with children's successful avoidance of goal neglect. These findings offer important insights into the cognitive and neural underpinnings of goal maintenance in preschoolers.
\end{abstract}

Keywords: goal maintenance, goal neglect, functional near-infrared spectroscopy, lateral prefrontal cortex, pre-schoolers 


\section{Cognitive and Neural Underpinnings of Goal Maintenance in Young Children}

\section{Introduction}

Children often fail to act in accordance with a goal despite correctly understanding what the goal is, and yet they gradually develop their ability to implement goal-directed behavior as opposed to automatic behavior. It has been repeatedly documented that this ability, known as executive functions, undergoes pronounced developmental improvements during early childhood (e.g., Best \& Miller, 2010; Diamond, 2013; Garon, Bryson, \& Smith, 2008; Zelazo et al., 2013). Although executive functions are composed of dissociable factors such as updating (i.e., holding and manipulating information in working memory), inhibition (i.e., suppressing prepotent responses), and shifting (i.e., switching flexibly between tasks or mental sets) (e.g., Miyake et al., 2000), active maintenance of task goal representations has been shown to be one of the common constructs among these factors in adults (Miyake \& Friedman, 2012) and a strong driver of the development of executive functions (Munakata, Snyder, \& Chatham, 2012). Thus, better understanding of goal maintenance functioning and its development can offer important insights into how children gradually develop executive functions.

As a conspicuous psychological phenomenon related to the malfunction of goal maintenance, previous studies have highlighted goal neglect, defined as a failure to execute momentary demands of a task due to temporary loss of the task goal from active memory, even though it is understood by the agent and still retrievable from long-term memory (Duncan, Emslie, Williams, Johnson \& Freer, 1996; Kane \& Engle, 2003). Goal neglect is typically accompanied by the inability to consistently hold goal representations in working memory, which has been evidenced by the finding that adults with low working memory capacity are more likely to neglect task goals (Kane \& Engle, 2003; McVay \& Kane, 2009). Moreover, 
patients with prefrontal cortex damage tend to neglect task goals when the environment lacks appropriate action prompts (e.g., Duncan, Burgess, \& Emslie, 1995; Duncan et al., 1996), suggesting that prefrontal cortical regions play a crucial role in maintaining such goal representations. Therefore, the study of goal neglect can provide information about the cognitive and neural mechanisms underlying goal maintenance. Further, pioneering work (Marcovitch, Boseovski, Knapp, \& Kane, 2010; Towse, Lewis, \& Knowles, 2007) has applied the method of inducing goal neglect used by adults in cognitive tasks for preschoolers. The present study employs such a method of assessing goal neglect in an attempt to elucidate the cognitive and neural underpinnings of goal maintenance in preschoolers.

\subsection{Cognitive underpinnings of goal maintenance in preschoolers}

It is well established that children often fail to maintain goal representations (Blackwell, Cepeda, \& Munakata, 2009; Chevalier \& Blaye, 2008; Marcovitch, Boseovski, \& Knapp, 2007; Marcovitch et al., 2010; Morton \& Munakata, 2002). For example, Marcovitch et al. (2007) conducted an experimental study to examine the role of goal neglect in the Dimensional Change Card Sort task (DCCS). In this task, children are introduced with the target bivalent cards using two dimensions (e.g., color: yellow and green, shape: cup and ship) and required to sort them according to one dimension (e.g., color, shape). In the color (or shape) game, children were instructed to place all of the yellow cards (or all of the cup cards) in the box with the yellow cup, and to place all of the green cards (or all of the ship cards) in the box with the green ship. In the pre-switch phase, the experimenter asked the participants to sort them according to one dimension. Once the pre-switch phase was completed, the post-switch phase began, and the experimenter provided the dimension switch instruction, that is, asked children to sort the test cards according to the other dimension. The DCCS requires two regulatory processes. One is 
conflict resolution, which is the process of overcoming prepotent but task-inappropriate responses (e.g., not sorting conflict cards according to a previously relevant dimension) and the other is goal maintenance, which is the process of maintaining task rules in working memory. Although the sorting performance on the DCCS reflects both conflict resolution and goal maintenance indistinguishably, an important characteristic of the goal neglect version of the DCCS is that it enables us to examine the role of maintaining goal representations by manipulating the need to keep task rules in mind. In the goal neglect version of the DCCS, redundant test cards, which match target cards in two dimensions, are interspersed with the standard conflict test cards. Redundant cards can be sorted without attending to the rules while conflict cards encourage awareness of the rules, thus goal maintenance is more challenging in the context of constantly sorting redundant cards than that of sorting conflict cards, which requires conflict resolution. When faced with a high proportion of redundant cards, children need to remind themselves to maintain the task rules endogenously to sort conflict cards. In contrast, when faced with a high proportion of conflict cards, the task environment helps children to keep the task rules active. Marcovitch et al. (2007) administrated two types of card decks that differed in their proportion of redundant cards to manipulate the demand on the maintenance of task rules. Children were more successful in sorting conflict cards in the mostly conflict deck $(80 \%$ conflict and $20 \%$ redundant cards) compared with the mostly redundant one (20\% conflict and $80 \%$ redundant). This finding suggests that the difficulty with maintaining task rules causes goal neglect (i.e., the occurrence of sorting errors).

To reveal cognitive underpinnings of goal maintenance, a large number of studies have examined whether working memory task performance accounts for goal maintenance (e.g., Engle \& Kane, 2004; Kane \& Engle, 2003; McVay \& Kane, 2009; Miller \& Cohen, 2001). Marcovitch 
et al. (2010) asked preschoolers to engage in three working memory tasks (i.e., visual counting span task, visual backward word span task, and auditory backward digit span task) and reported that their working memory performance was associated with the performance on both types of decks of the goal neglect DCCS, suggesting that whether young children can maintain goal representations and resolve conflicts heavily depends on their working memory functioning. In addition, a recent study with preschoolers using a different experimental paradigm (Gonthier, Zira, Colé, \& Blaye, 2019), found a direct link between performance on two working memory tasks (backward color recall task, block tapping task) and the ability to keep goal relevant information in an active state. They asked 4-7-year-olds to perform the AX Continuous Performance Task (AX-CPT) (Braver et al., 2001; Chatham, Frank, \& Munakata, 2009), which was designed to assess whether participants can continuously hold contextual information (e.g., $\mathrm{X}, \mathrm{Y}$ ) to prepare probes (e.g., A, B) requiring next responses in advance (proactive control) or retrospectively remember contextual information just after the appearance of the stimuli (reactive control). This study confirmed that age-related increase in the use of proactive control is explained by the development of working memory performance. These developmental findings suggest that in preschoolers, working memory capacity underlies the ability to hold task goals and goal relevant information.

However, considering the psychological constructs measured by working memory tasks carefully, there is a possibility that working memory performance might partially reflect inhibitory control. Hasher, Lustig, and Zacks (2007) provided much empirical effort to understand inhibitory control as a critical source of working memory variations from the study of age-related inhibitory control deficits and intra-individual differences. From the perspective of executive functions (Miyake \& Friedman, 2012), working memory updating tasks capture 
updating-specific factors, but they are also explained sufficiently by a factor extracted from inhibition tasks, suggesting that it is possible that working memory updating tasks actually measure inhibitory control and working memory that remains controlling for inhibitory control. In a similar vein, Engle and Kane (2004) suggested that working memory tasks measure both goal maintenance and conflict resolution. At least, the regulatory process of conflict resolution is conceptually similar to that of inhibitory control. From the developmental perspective, although a number of studies supported the validity of a unitary factor model in young children (e.g., Hughes, Ensor, Wilson, \& Graham, 2010; Wiebe, Espy, \& Charak, 2008), there are pieces of evidence supporting a two-factor model of executive functions consisting of working memory updating and inhibitory control factors that are closely related but can be separable (Lerner \& Lonigan, 2014; Miller, Giesbrecht, Müller, McInerney, \& Kerns, 2012; Usai, Viterbori, Traverso, \& De Franchis, 2014). Engle and Kane's account is consistent with the abovementioned findings in young children (Marcovitch et al., 2010). Furthermore, Marton, Kelmenson, and Pinkhasova (2007) demonstrated that working memory tasks are highly demanding on inhibitory control in childhood, which is evidenced by the finding that working memory capacity can be enhanced by increasing the efficiency of inhibitory control through the comparison between typical children and children with specific language impairment. Therefore, if we define only working memory tasks as predictors of goal maintenance, it remains unclear how inhibitory control influences goal maintenance. Thus, the current study conducted both working memory tasks and inhibitory control tasks in order to clarify each unique effect of working memory capacity and inhibitory control on goal maintenance.

One of the possible roles of inhibitory control in goal maintenance is to help children suppress currently irrelevant information that interferes with the goals. Indeed, goal 
representations must be not only kept in an active state but also shielded from interference (Dreisbach \& Goschke, 2004). For example, in performing the DCCS task, children should inhibit a formerly relevant sorting rule to activate a newly relevant sorting rule (Zelazo, Müller, Frye, \& Marcovitch, 2003). It is also the case that children should ignore the stimulus feature of the irrelevant dimension to resist distracting interference. Consistent with the assumption that goal maintenance is supported by inhibitory control, Towse et al. (2007) showed that goal neglect, which is measured by the tendency to neglect visual cues that direct to one or another stimuli stream, was uniquely linked to the inhibitory control abilities of preschool children. This result suggests that the failure in inhibiting the responses to goal-irrelevant stimuli promotes the neglection of task goals.

Therefore, we expected that both working memory capacity and inhibitory control are potential factors underlying the active maintenance of goal representations in preschoolers. Specifically, working memory seems to play a role in keeping task goals in an active state over time, while inhibitory control protects task goals through suppression of previously relevant representations/responses or goal-irrelevant stimuli. Indeed, Chevalier et al. (2012) showed that both working memory capacity and inhibitory control can uniquely account for mixing costs, which are considered to reflect the ability to maintain two task-sets and monitor when to switch task-sets under the uncertain condition where switching may happen, in a paradigm for measuring cognitive flexibility. However, to the best of our knowledge, few studies have directly examined whether both working memory capacity and inhibitory control contribute to goal maintenance, using the paradigm for assessing goal neglect. In this work, we employed the goal neglect DCCS task (Marcovitch et al., 2010) and predicted that lower working memory capacity and inhibitory control would lead to more errors in sorting conflict cards while dealing with the 
mostly redundant deck. Specifically, we aimed to examine how working memory capacity and inhibitory control uniquely account for the sorting performance.

\subsection{Neural underpinnings of goal maintenance in preschoolers}

In adult participants, a growing body of research has been gathered showing that goal maintenance is linked with the activation of dorsolateral regions of the prefrontal cortex in a variety of cognitive tasks (e.g., Braver \& Cohen, 2001; Lopez-Garcia et al., 2016; MacDonald \& Cohen, 2003; MacDonald, Cohen, Stenger, \& Carter, 2000; Paxton, Barch, Racine, \& Braver, 2007). For example, in the Stroop task adult participants were instructed to read a word or name a color before each trial, but during the instruction period, in which participants were simply waiting for the start of the task, the left dorsolateral prefrontal regions were more active to name the color than read the word. In addition, increased prefrontal activation during the instruction phase was associated with smaller interference effects when responding to incongruent colored words (MacDonald et al., 2000). In the AX-CPT task, Braver and Cohen (2001) established that the activity of the dorsolateral prefrontal areas increases when the necessary duration of maintaining goal relevant information increases, by comparing activity between short delay and long delay trials. They also found that the pattern of activity in the left dorsolateral prefrontal region shows disruption under conditions of interference. These findings succeeded in separating goal maintenance from other cognitive processes, demonstrating that dorsolateral prefrontal regions are found to play a crucial role in maintaining task goals or goal relevant information in working memory.

For preschoolers, to the extent of our knowledge of the literature, no study has directly examined the neural mechanisms underlying goal maintenance. Recent neuroimaging studies for preschoolers have used functional near-infrared spectroscopy (fNIRS) (Mehnert et al., 2013; 
Moriguchi \& Hiraki, 2009, 2011; Perlman, Huppert, \& Luna, 2016; Smith et al., 2017; Tsujimoto, Yamamoto, Kawaguchi, Koizumi, \& Sawaguchi, 2004; for a review, Fiske \& Holmboe, 2019; Moriguchi \& Hiraki, 2013). fNIRS uses safe, non-invasive near-infrared light for the assessment of brain function, and a cap containing sources and detectors of the nearinfrared light is placed on the participant's head. Unlike functional magnetic resonance imaging (fMRI), fNIRS is restraint-free and it is relatively robust with respect to movement. This makes the fNIRS attractive for use with young children to examine their neurodevelopmental mechanism (Fiske \& Holmboe, 2019). The validity of fNIRS has been confirmed by previous studies on the prefrontal regions in adults (e.g., Hermann \& Fallagatter, 2003; Sumitani et al., 2006) and in children (e.g., Moriguchi \& Hiraki, 2009, 2011; Perlman et al., 2016; Tsujimoto et al., 2004). For instance, Sumitani et al. (2006) demonstrated that adults recruited lateral prefrontal activation during a cognitive shifting task, namely the Wisconsin Card Sorting Test (WCST). In a study with children three to seven years old, Perlman et al. (2016) also found a strong activation of the lateral prefrontal regions in the test phase of a spatial working memory task, which was positively correlated with child age. However, the previous developmental work using fNIRS have not identified the neural bases of goal maintenance by strictly separating the cognitive process underlying goal maintenance from the other regulatory processes. For instance, Moriguchi and Hiraki $(2009,2011)$ found that children who could switch rules perfectly in the DCCS activated inferior prefrontal areas during the pre-switch and post-switch sessions. It is possible that the prefrontal activation would reflect successful conflict resolution in sorting conflict cards according to a rule and/or reflect the maintenance of the task goal, as Moriguchi and Hiraki $(2009,2011)$ did not directly examine whether children recruit the prefrontal regions in the active maintenance of goal representations by, for example, manipulating the proportion of 
redundant cards as in Marcovitch et al. (2007, 2010). We, therefore, aimed to measure prefrontal activation underlying goal maintenance by using the aforementioned paradigm for measuring goal neglect (Marcovitch et al. 2007, 2010).

\subsection{Current study}

In the current study, we modified the original goal neglect DCCS task for measuring the neural activity. Although the original goal neglect DCCS requires children to sort both the conflict cards and the redundant cards within one block, the current study set up a conflict (or redundant) block in which children sort conflict (or redundant) cards consecutively, resulting in children experiencing the conflict block and the redundant block alternatively with a short rest (a rest phase). Specifically, as shown in Figure 1, after sorting conflict cards in the pre-switch session, children work on the post-switch session in the revised goal neglect DCCS, which is composed of the first conflict block, the redundant block, and the second conflict block. In the first conflict block, children sort conflict cards consecutively according to the post-switch rule, and then participate in the redundant block, in which they sort redundant cards consecutively. Finally, in the second conflict block, they sort conflict cards again. It is reasonable to assume that the redundant block, in which goal maintenance is challenging, should place a high demand on holding the post-switch rule in mind for the second conflict block, resulting that the sorting performance in the second conflict block would decline. In terms of the neural activity, we expect that the activation in prefrontal regions during the two conflict blocks and the redundant block should reflect different psychological processes depending on the type of sorted test cards. In particular, we assume that the prefrontal activation in the conflict blocks should mainly reflect goal maintenance and conflict resolution, whereas the prefrontal activation in the redundant blocks should mainly reflect goal maintenance. It is also assumed that children can sort 
redundant cards without maintaining a post-switch rule by perceptually matching the test card with the target cards. Yet, children who rely on the perceptual-matching strategy would not exhibit the prefrontal activation in the redundant block, thus the prefrontal activation while sorting consecutive redundant cards can be interpreted as reflecting goal maintenance.

Importantly, we expected that the prefrontal activation during the redundant block session should predict the performance on the second conflict block, because the sorting performance in the second conflict block depends on whether children can keep a rule in mind.

(Please insert Figure 1 about here)

Taken together, the current study required children to perform the original goal neglect DCCS task (Marcovitch et al., 2010), the revised goal neglect task, two working memory tasks, and two inhibition tasks. We aimed to: 1) replicate the findings from the original goal neglect DCCS using the same procedure as Marcovitch et al. (2010), 2) examine how working memory capacity and inhibitory control associate with performance in both the original and the revised goal-neglect DCCS, 3) test whether children would activate prefrontal regions while sorting cards in the revised goal neglect DCCS, and 4) examine whether the activation in prefrontal regions while sorting redundant cards could be associated with the avoidance of goal neglect in the second conflict block of the revised goal neglect DCCS. Our key predictions were: 1) regarding the original goal neglect DCCS, children should be more successful in sorting conflict cards while working with a deck mostly composed of conflict cards rather than a deck mostly composed of redundant cards, 2) the performance of inhibition tasks should predict the performance of the goal neglect DCCS in the mostly conflict card deck. Moreover, the 
performance on working memory tasks and inhibition tasks should predict the performance of the goal neglect DCCS in the mostly redundant deck. We further hypothesize that: 3) children should activate prefrontal regions more during the task phase than the rest phase in the preswitch session, the first conflict block, and the redundant block, and 4) the activation in prefrontal regions during a task phase in the redundant blocks should predict the performance of sorting conflict cards in the second conflict blocks.

\section{Method}

The study design, hypotheses, and analytic plan were preregistered in the Open Science Framework (https://osf.io/e6qh3/).

\subsection{Participants}

To determine the sample size, we referred to Marcovitch et al. (2010) reporting a significant main effect of deck type condition (mostly conflict condition versus mostly redundant condition) with an effect size of $\eta^{2}{ }_{p}=.10$ from 65 children. According to our preregistered estimation, at least 38 children were required to have enough power to detect the same effect size. ${ }^{1}$ However, in terms of the relation between behavioral data and the prefrontal activation, it seems difficult to determine the appropriate sample size due to the exploratory nature of this study. Thus, in terms of collecting both behavioral and neural data, we decided on a sample size of 50 children. Considering that several children may refuse to wear the fNIRS probe and that some data may have been unavailable due to unstable measurement, it was expected that we needed to collect information from 10 more children than the planned sample size. Participants were 61 children ( 28 boys and 33 girls; $M=58.7$ months, $S D=9.2$, age range $=46-79$ months) attending preschool in Japan. Among the children who were tested, six refused to wear the 
fNIRS probe, but they engaged in other behavioral tasks. We therefore report behavioral data from 61 children and neural data from 55 children $(25$ boys and 30 girls; $M=59.6$ months, $S D=$ 8.8 , age range $=50-79$ months). However, several children did not show stable neural data for one or some of our target channels (i.e. ch4, ch5, ch13, and ch14) of fNIRS. They were excluded from the analysis for each channel (e.g., ch4). Therefore, the number of the final sample was roughly as expected. None of the participants had any history of neurological disorders, and all of them were native Japanese speakers. Their socioeconomic background was predominantly middle class. Regarding research ethics, we gave school staff members a brief explanation about the aim of the current study, the function of fNIRS, and its safety and obtained their consent for conducting this experiment in the school. We obtained informed consent from the parents for all children prior to their participation. This study was conducted in accordance with the principles of the Declaration of Helsinki and approved by the institutional ethics committee for experimental psychology research at Graduate School of Education, Kyoto University (approval number: CPE-228; title: 'Neural bases of goal neglect in young children: a near-infrared spectroscopy (fNIRS) study').

\subsection{Procedure}

All participants were tested in the two deck types of the original goal neglect DCCS, the revised goal neglect DCCS, and the following four tasks, which are often used for assessing working memory capacity or inhibitory control. In line with Marcovitch et al. (2010), as measures of working memory capacity, backward digit span task and visual counting span task were selected. The backward digit span task mainly involved a verbal domain and the visual counting span task mainly involved a visual domain. Similarly, we selected one inhibition task that mainly depends on a visual domain and another inhibition task mainly depends on a verbal 
domain. Thus, we decided to employ the fish flanker task and the red/blue task to measure inhibitory control. The former inhibitory control task relies more on a visual domain, whereas the latter inhibitory control task is more related to a verbal domain. These tasks were tested over two days. On the first day, children completed the revised goal neglect DCCS, one deck type of the original goal neglect DCCS, and one of the two working memory and two inhibition tasks. On the second day, children completed the other deck type of the original goal neglect DCCS and the other three tasks. The order of two working memory and two inhibition tasks was counterbalanced across participants. To put the children at ease, both sessions were conducted in a quiet room at the preschool. The testing required approximately twenty minutes per session.

\subsection{Measures}

\subsubsection{Original goal neglect DCCS.}

This task was conducted to measure the extent to which children neglected goal representations through the comparison of responses in the mostly redundant deck and the mostly conflict deck. First, the children were presented with two trays and two target cards, and the experimenter labelled the target cards using two dimensions (yellow and green, cup and ship). Then, the experimenter introduced the redundant test cards, which were same as the target cards, and conflict test cards, sharing a feature of one dimension with the target cards. In the preswitch session, using only the conflict cards, 10 test cards were sorted according to the relevant rule (color or shape) in a pseudo-random sequence, and no feedback was provided. For example, while sorting test cards according to the shape rule, children had to place all the cup cards in the box with the yellow cup, and place all the ship cards in the box with the green ship. The procedures of introducing cards and the pre-switch session were same in both the mostly redundant deck and the mostly conflict deck. We used the same criterion as used in Marcovitch 
et al. (2007), which stated that the children who correctly sorted more than eight cards were included in the final sample. As a result, all the participants could meet the criterion and were included in the final sample.

Once the pre-switch session was completed, the experimenter provided the post-switch session instruction, that is, introducing the children to a new game in which they were instructed to sort the test cards according to the opposite dimension. In the post-switch session, the children were presented with 30 test cards and were asked to sort them according to the new rule, and the rule was repeated every fifth trial. In the mostly redundant deck, the redundant cards were used in the first six trials and 18 of the remaining 24 trials and the conflict cards were used in the other trials, that is, $80 \%$ of all post-switch trials used redundant cards. The redundant cards were interspersed with the conflict cards not to appear consecutively. In the mostly conflict deck, this contingency was reversed, that is, $80 \%$ of all post-switch trials used conflict cards. The sorting rule was counterbalanced across participants. The materials (i.e., features of the dimensions) are different between the mostly conflict deck and the mostly redundant deck. The dependent variable was the proportion of conflict cards sorted correctly during the post-switch session.

\subsubsection{Backward digit span task.}

This task was administrated to measure working memory capacity. The stimuli were digits 1-9 that were prerecorded and presented auditorily via computer at a rate of 1 per second. At the beginning of each trial, children were instructed to recall the sequence in reverse order. No digit could appear twice in the same trial. A practice trial was administrated with a two-digits list, and if they correctly performed the trial, they proceeded to the test phase. If the children failed, they were given the instruction again until they answered correctly. In the test phase, if the children perfectly recalled more than one trial at each length sequence, they proceeded to the next length 
sequence. When they could not correctly recall any trials at a given sequence length, the testing ended. Each sequence length included three trials, ranging from sequence lengths two to five. During the test trials, no feedback was provided. Following Conway, Kane, Bunting, Hambrick, Wilhelm, and Engle (2005), across all trials, we calculated the sum of the proportion of elements recalled in correct serial position within each trial.

\subsubsection{Visual counting span task.}

This task was also conducted to measure working memory capacity, and was originally designed by Case, Kurland, and Goldberg (1982). In this task, several sheets, on which two types of objects (frogs and cats, stars and rainbows, or suns and hearts) were depicted, were placed face down on a desk. One sheet at a time was turned over by the experimenter and the children were asked to count one type of the object (e.g., frog) before turning the sheet face down. After all the sheets were counted, they had to recall the counts of each set in the correct order. First, they started with a practice trial, in which they had to count and recall two sheets. In the test phase, there were three trials at each span level (two, three, four, and five span levels). As with the backward digit span task, if the children perfectly recalled even one trial in the correct order, they went to the next span level; otherwise, the testing ended. During the test trials, no feedback was provided. The procedure of the scoring was same as that of the backward digit span task.

\subsubsection{Fish flanker task.}

This task was conducted to measure the children's inhibitory control and was developed by Rueda et al. (2004) as a child-friendly version of the Eriksen flanker task. We administrated this task with almost the same procedure as the fish block in the flanker task developed by Zelazo, Anderson, Richler, Wallner-Allen, and Beaumont (2013). In this task, five fish appeared on the screen of a laptop computer (Surface Pro 4; Microsoft), and the children were asked to judge 
which direction a middle fish was facing while ignoring two fish on either side that were facing the congruent direction or the incongruent direction with the target fish. At the beginning of the task, they were required to answer which fish was centrally positioned and press one of two buttons (i.e., a right and left arrow) according to the directions of the middle fish. They were also given an explanation about the two conditions where four fish flanking the middle fish were either facing the congruent direction or the incongruent direction. In the practice phase, they were given two congruent trials and two incongruent trials with feedback, and this phase was repeated until they passed all the four trials. In the test phase, they received 10 congruent and 10 incongruent trials presented in a pseudorandom order. As the dependent measure, we calculated the difference between the response time for congruent trials and incongruent trials, due to the ceiling performance. We excluded the trials in which children answered incorrectly from the analysis.

\subsubsection{Red/blue task.}

This task was also conducted to assess the children's inhibitory control. The procedure was essentially the same as that of the black-white task used by Simpson and Riggs (2005). First, the children were asked to point to a blue card with one hand when the experimenter said "blue" and to point to a red card with one hand when he said "red." All participants were able to answer correctly. Then, children were asked to point to the red card when the experimenter said "blue" and to the blue card when he said "red." Two practice trials were given to the children, and if they responded correctly to the two trials in succession, they proceeded to the test phase. If they answered incorrectly in the practice phase, the task instruction was repeated. This procedure was repeated until they correctly answered the two practice trials in succession. In the test phase, children engaged in 16 trials, during which the experimenter said "red" and "blue" eight times at 
a pace of one trial per second, each in a pseudo-random sequence. The task rule was not repeated and no feedback was provided. The dependent measure was the number of correct trials during a session (0-16).

\subsubsection{Scoring and standardization of working memory tasks and inhibition tasks}

In terms of working memory tasks (backward digit span task, visual counting span task) and inhibition tasks (fish flanker task, red/blue task), we created composite scores of working memory capacity and inhibitory control by summing each standardized working memory and inhibition measures to minimize the influence of task-specific variance, leading to better measures as individual-differences constructs. This scoring procedure was consistent with Marcovitch et al. (2010).

\subsubsection{Revised goal neglect DCCS.}

This task was developed revising the original goal neglect DCCS for measuring activity in the prefrontal region. At the beginning of this task, the fNIRS equipment was securely placed upon the child's head. This task was also composed of both pre-switch and post-switch sessions (Figure 1). In the pre-switch session, children sat still in a rest phase (35 seconds) and sorted conflict cards (20 seconds) according to the first rule (e.g., color) in a task phase. The maximum number of conflict cards was ten and 9.29 cards were sorted on average during the task phase. Next, in the post-switch session, including the first conflict block, the redundant block, and the second conflict block, children were asked to sort cards according to the second rule (e.g., shape). In the first conflict block, children sat still in a rest phase (35 seconds) and sorted conflict cards consecutively in a task phase (20 seconds). The maximum number of conflict cards was also ten and children sorted 9.42 cards on average. Next, they participated in the redundant block, in which they sat still in a rest phase (35 seconds) and sorted redundant cards 
consecutively in a task phase (20 seconds). The maximum number of redundant cards was also ten and children sorted 9.68 cards on average. Finally, without a rest phase, the experimenter reminded children of the post-switch rule and they proceeded to sort 6 conflict cards in the second conflict block. We did not insert a rest phase at the beginning of the second conflict block because it would have been difficult for us to examine whether active maintenance of goal representations during redundant sort directly affects subsequent conflict sort. Test cards were handed over at the experimenter's pace in order that children continued to sort test cards throughout the task phase. Therefore, there were not any cases where children finished earlier and just waited. All of the participants sorted six test cards or more in each task phase. The number of test cards children sorted in the first conflict block was not associated with the sorting performance $(r(55)=-.017, p=.631)$. One cycle, in which children engaged in the pre-switch phase and then in the post-switch phase, was repeated three times using different materials (i.e., features of the dimensions). This procedure followed that of Moriguchi and Shinohara (2018), in which preschoolers underwent three consecutive test sessions with the DCCS task. The dependent variable was the proportion of conflict cards sorted correctly during the first conflict and the second conflict block.

\subsection{8. fNIRS recordings.}

During the revised goal-neglect DCCS, we employed a multichannel fNIRS unit operating at wavelengths of 770 and $840 \mathrm{~nm}$ (OEG-16; Spectratech Inc., Tokyo, Japan). The 16 channel fNIRS probe included 12 optodes, and the probes were placed on the lateral prefrontal areas of each hemisphere. The temporal resolution at each channel was of approximately $666 \mathrm{~ms}$.

As shown in Figure 2, the center of the fNIRS probe was placed on the International 10/20 system, and the bottom left and right corners were located around F3 and F4. The spatial 
resolution of fNIRS is relatively low, thus we aggregated channels 2,4 , and 5 into the right lateral prefrontal region and channels 11,13, and 14 into the left lateral prefrontal regions, which were corresponding F4 and F3 (Okamoto et al., 2004). Previous studies (Moriguchi \& Hiraki, 2009; Moriguchi \& Shinohara, 2018) confirmed the activation of these areas during the test phase of the DCCS. Thus, it is highly likely that fNIRS system is effective for measuring activation in the lateral prefrontal activation. In the current study, due to a problem with the probe, we could not collect enough neural data for channel 2 and 11 to conduct analyses; thus, we report the results of channel 4, 5, 13, and 14 from 55 children. Given the lateral spatial resolutions of fNIRS, each channel partially corresponds to the lateral prefrontal cortex and the exclusion of channel 2 and 11 has little effect on our main focuses and analyses.

fNIRS measurements are indexed by temporal changes in the concentrations of oxygenated hemoglobin (oxy-Hb) and deoxygenated hemoglobin (deoxy-Hb). For each subject, the average changes in oxy- $\mathrm{Hb}$ and deoxy- $\mathrm{Hb}$ during the rest and the task phases were calculated for each channel. First, the data were filtered with the moving average, and then baseline correction was performed using linear fitting. The fNIRS signal was separated into both functional (i.e., brain activation) and systematic (i.e., physiological noise) components. Several methods have been proposed for separating only the functional components from all the rest of components (Huppert, Diamond, Franceschini, \& Boas, 2009). For example, a signal correlation method (e.g., Saager, Telleli, \& Berger, 2011) is useful to reduce baseline fluctuations or irregular motion artifacts, while it is not effective for removing systematic fluctuations, because this method assumes that there is no correlation between functional and systematic components. We, therefore, adapted a recently proposed method (Yamada, Umeyama, \& Matsuda, 2012), in which functional components are assumed to be based on a negative relation between oxy-Hb and 
deoxy-Hb changes and systematic components are based on a positive relation, and we only used the functional components.

(Please insert Figure 2 about here)

\section{Results}

Final data files for this study are available via the Open Science Framework (https://osf.io/e6qh3/).

\subsection{Descriptive statistics}

Descriptive statistics for children's performance on the original goal neglect DCCS, backward digit span task, visual counting span task, red/blue task, and fish flanker task are provided in Table 1. The results of correlational analysis are also summarized in Table 1. Cronbach's alpha was computed as an index of internal consistency for the working memory tasks and one of the inhibition tasks. Reliability analyses revealed Cronbach's alpha of 0.80 (backward digit span task), 0.87 (visual counting span task), and 0.60 (red/blue task), indicating medium or high internal consistency. For the fish flanker task, spilt-half consistency between interference computed on the odd-numbered and even-numbered trials using Spearman-Brown adjustment was 0.70 , which also indicated medium internal consistency. 
Table 1

Correlation among the goal neglect DCCS, two working memory tasks, and two inhibition tasks

\begin{tabular}{|c|c|c|c|c|c|c|}
\hline & $\begin{array}{l}\text { Goal neglect } \\
\text { DCCS } \\
\text { /Redundant } \\
\text { deck }\end{array}$ & $\begin{array}{l}\text { Goal neglect } \\
\text { DCCS } \\
\text { /Conflict } \\
\text { deck }\end{array}$ & $\begin{array}{l}\text { Digit span } \\
\text { (correct } \\
\text { trials) }\end{array}$ & $\begin{array}{l}\text { Counting } \\
\text { span } \\
\text { (correct } \\
\text { trials) }\end{array}$ & $\begin{array}{l}\text { Fish flanker } \\
\begin{array}{c}\text { (response } \\
\text { time) }\end{array}\end{array}$ & $\begin{array}{c}\text { Red/blue } \\
\text { (correct trials) }\end{array}$ \\
\hline Range & $0-1$ & $0-1$ & $3.5-11.2$ & $3.66-12$ & $\begin{array}{c}-91-622 \\
(\mathrm{~ms})\end{array}$ & $8-16$ \\
\hline Mean (SD) & $0.65(0.36)$ & $0.91(0.19)$ & $\begin{array}{c}6.39 \\
(1.73)\end{array}$ & $\begin{array}{c}7.05 \\
(2.34)\end{array}$ & $\begin{array}{l}150.95 \\
(179.6)\end{array}$ & $\begin{array}{l}13.74 \\
(1.91)\end{array}$ \\
\hline \multicolumn{7}{|l|}{ Correlation } \\
\hline $\begin{array}{l}\text { Goal neglect } \\
\text { DCCS } \\
\text { (Redundant deck) }\end{array}$ & - & - & - & - & - & - \\
\hline $\begin{array}{l}\text { Goal neglect } \\
\text { DCCS } \\
\text { (Conflict deck) }\end{array}$ & $.48 * * *$ & - & - & - & - & - \\
\hline Digit span & $.37 * *$ & $.41^{* *}$ & - & - & - & - \\
\hline Counting span & $.26^{*}$ & $.34 *$ & $.36^{* *}$ & - & - & - \\
\hline Fish flanker & $-.30^{*}$ & -.10 & $-.34^{*}$ & $-.27 *$ & - & \\
\hline Red/blue & $.68 * * *$ & $.59 * * *$ & $.42 * * *$ & $.42 * * *$ & -.20 & - \\
\hline Age in months & $.33 * *$ & .23 & $.54 * * *$ & $.54 * * *$ & $.44 * * *$ & $-.35^{* *}$ \\
\hline
\end{tabular}

${ }^{*} p<.05, * * p<.01, * * * p<.001$

\subsection{Behavioral results in the original goal neglect DCCS}

Children performed nearly perfectly in sorting redundant cards $(97 \%)$. Thus, we conducted a series of hierarchical multiple regressions with the proportion of correctly sorted conflict cards in the original goal neglect DCCS task as a dependent variable, using the R system for statistical computing (R Core Team, 2013). First, it was aimed to replicate the findings from the original 
goal neglect DCCS (Marcovitch et al., 2007). We examined whether the correct rates differed between in the mostly conflict deck and in the mostly redundant deck and the difference between the two types of decks interacted with age in months. We also controlled the influence by the presentation order of the two deck types and that of the two dimensions. As a first step, a factor of deck type (mostly conflict deck $=1$, mostly redundant deck $=-1$ ), a factor of age in months, as well as the interaction between the two factors, the order of deck types (mostly conflict deck $\rightarrow$ mostly redundant deck $=1$, mostly redundant deck $\rightarrow$ mostly conflict deck $=-1)$ and the order of two dimensions (color $\rightarrow$ shape $=1$, shape $\rightarrow$ color $=-1$ ) were entered into the regression model as a predictor variable. The age factor was centered. Second, to replicate and extend the findings from Marcovitch et al. (2010), we examined how working memory capacity and inhibitory control associated with the performance of the original goal neglect DCCS task beyond age differences (a factor of age in months). As a second step, factors of standardized working memory capacity and inhibitory control along with their interactions with the deck type factor were added to the above model.

At the first step, the regression model, which included age in months, deck types, the order of deck types, and the presentation order of the two dimensions, revealed significant main effects of age in months $(b=0.009, S E=0.003, t=2.92, p=.004)$ and deck type factor $(b=0.40, S E=$ $0.19, t=2.14, p=.034)$. These findings indicated that older children performed better and children sorted conflict cards more correctly in the mostly conflict deck than in the mostly redundant deck (see Figure 3). There was no interaction between age in months and deck type ( $b$ $=-0.004, S E=0.003, t=-1.48, p=.143)$. Next, at the second step, in which factors related to standardized working memory capacity and inhibitory control were added, the results of the full model are summarized in Table 2. Inhibitory control was a significant factor, and working 
memory capacity was a marginal significant factor. In contrast, a main effect of age in months changed to be non-significant when factors of working memory capacity and inhibitory control were entered. Importantly, we found a significant interaction between inhibitory control and deck type. According to the post-hoc analysis, the inhibition task performance was a significant factor $(b=.12, t=4.83, p<.001)$ in the mostly redundant deck, and it was not significant in the mostly conflict deck ( $b=.04, t=1.57, p=.118)$. In addition, the results of another regression model including age-related interactions with working memory capacity and inhibitory control can be seen in Appendix A, indicating no significant age-related interactions.

(Please insert Figure 3 about here)

Table 2

Results of regression analysis examining the effects of working memory capacity, inhibitory control, and sort deck type on the performance of the original goal neglect DCCS

\begin{tabular}{ccccc} 
Fixed effect & Estimate & $S E$ & $t$ value & $\operatorname{Pr}(>|t|)$ \\
\hline (Intercept) & 0.786 & 0.02 & 35.99 & $<.001$ \\
Age in months & -0.003 & 0.004 & -0.69 & .491 \\
Order (dimension) & 0.015 & 0.022 & 0.64 & .525 \\
Order (condition) & -0.006 & 0.022 & -0.26 & .793 \\
Deck type & 0.129 & 0.022 & 5.93 & $<.001$ \\
WM_composite & 0.033 & 0.018 & 1.81 & .074 \\
Deck type $\times$ WM_composite & -0.001 & 0.016 & 0.94 & .938 \\
Inhibit_composite & 0.079 & 0.018 & 4.47 & $<.001$ \\
Deck type $\times$ Inhibit_composite & -0.040 & 0.017 & -2.33 & .022 \\
\hline
\end{tabular}


Note: "Order (condition)" indicates the condition in which the participants performed the tasks first, and "Order (dimension)" indicates the dimension of the performed sorting of cards, colors, and shapes. Additionally, both "WM_composite" and "Inhibit_composite" are composite scores generated by standardizing working memory capacity and inhibition measures.

We noticed that there were the positive correlations between the red/blue task and the performance on both sorts, but the fish flanker task significantly correlated only with the performance on the mostly redundant deck but not with that on the mostly conflict deck (see Table 1). Although we initially categorized these two tasks together into one task group, it is possible that the two inhibitory tasks were differently associated with the two types of sorts in the original goal neglect DCCS. Therefore, we separated the composite score of inhibitory control into a factor of the red/blue task and a factor of the fish flanker task and examined differences in the impact of the red/blue task and the flanker task on the sorting performance of the original goal neglect DCCS. Instead of a main effect of the composite score of inhibitory control and its interaction with a deck type factor, we added main effects of red/blue task performance and flanker task performance and their interactions with a deck type factor to the aforementioned model, although this is a post-hoc analysis. It was shown that a main effect of the $\mathrm{red} / \mathrm{blue}$ task was significant $(b=0.14, S E=0.02, t=5.71, p<.001)$, but that of the fish flanker task was not significant $(b=0.03, S E=0.02, t=1.11, p=.268)$. In addition, there was a marginal significant interaction between the fish flanker task and deck type $(b=-0.04, S E=0.02$, $t=-1.77, p=.080)$, but the red/blue task did not interact with the deck type factor $(b=-0.04, S E$ $=0.02, t=-1.65, p=.103)$. Although the interaction between the fish flanker task and deck type was insufficient, simple effects revealed that fish flanker task performance was a significant factor $(b=.07, t=2.03, p=.044)$ in the mostly redundant deck, and it was not significant in the mostly conflict deck $(b=-.01, t=-0.45, p=.652)$. 


\subsection{Behavioral results in the revised goal neglect DCCS}

To confirm whether children neglected goal representations in the revised goal neglect DCCS, we initially compared the proportion of correctly sorted conflict cards in the first conflict block with that of the second conflict block. As children repeatedly performed the same task three times using different materials, the data were analyzed in each cycle with generalized linear mixed-models (GLMMs) using the lme4 package (Bates, Maechler, Bolker, \& Walker, 2015) in the R system. A regression model, which included block type (within: first conflict $=1$, second conflict $=-1$ ) and the presentation order of the dimensions as fixed-effect variables, was conducted with the proportion of sorting conflict cards as a dependent variable. For the random effects, a random intercept for participants was included. We identified significant main effects of block type $(b=0.08, S E=0.01, t=6.16, p<.001)$, which indicated that children had more difficulty with sorting conflict cards in the second conflict block (Mean $=0.79, S D=0.35)$ than in the first conflict block (Mean $=0.94, S D=0.18)$.

When targeting the complete sample, incorrect sorting in the second conflict block is contaminated by the incorrect performance of the first conflict block. During the first conflict block, the failure to resolve conflict and maintain a post-switch rule can potentially cause children to sort conflict cards according to a pre-switch rule. When children fail in the first conflict block, they are likely to respond incorrectly in the second conflict block regardless of the influences from the redundant block. The children's failure in sorting conflict cards in the second conflict block can be attributed to their failure to resolve conflict and maintain a post-switch rule in the first conflict block, rather than their difficulty with maintaining goal representations when sorting redundant cards. Therefore, hereafter, in analyzing the performance on sorts in the second 
conflict block, the cycles in which children could not sort perfectly in the first conflict block were excluded. Thus, targeting cycles in which children could sort conflict cards perfectly in the first conflict block ${ }^{2}$, we examined how working memory capacity and inhibitory control were associated with the performance of the revised goal neglect DCCS beyond age differences. Our analysis included the factor of age in months, the order of presentation of the two dimensions, working memory capacity, and inhibitory control as fixed-effect variable. For the random effects, a random intercept for the participant was also included. Table 3 shows the final model for the performance on sorts in the second conflict block. According to the model, we identified a significant main effect of inhibitory control, indicating that inhibitory control was related to the performance of sorting conflict cards in the second conflict block. Moreover, working memory capacity was found to be a marginal significant factor. These results exhibit a similar pattern as those of the original goal neglect DCCS.

Table 3

Results of regression analysis examining the effects of working memory capacity and inhibitory control on the performance of the revised goal neglect DCCS

\begin{tabular}{ccccc}
\hline Fixed effect & Estimate & $S E$ & $t$ value & $\operatorname{Pr}(>|t|)$ \\
\hline (Intercept) & 0.824 & 0.026 & 31.88 & $<.001$ \\
Age in months & 0.004 & 0.004 & 0.99 & .326 \\
Order (dimension) & 0.017 & 0.027 & 0.63 & .534 \\
WM_composite & 0.039 & 0.021 & 1.82 & .074 \\
Inhibit_composite & 0.048 & 0.021 & 2.29 & .026 \\
\hline
\end{tabular}

Note: "Order (dimension)" indicates the dimension of the performed sorting of cards, colors, and shapes. Both "WM_composite" and "Inhibit_composite" are composite scores generated by standardizing working memory capacity and inhibition measures. 


\section{4. fNIRS results in the revised goal neglect DCCS}

We tested whether children would activate lateral prefrontal regions while sorting redundant cards as well as conflict cards in the revised goal neglect DCCS. Main analyses did not include the cycle in which the number of sorted test cards were below three standard deviations from the average. The minimum number of sorted test cards for inclusion in the main analyses was seven. Six cycles in the pre-switch session, four cycles in the first conflict block, and no cycles in the redundant block were excluded. After separating the fNIRS signal into functional and systematic components, the results for the oxy- $\mathrm{Hb}$ and deoxy-Hb were consistent. Thus, we report the oxy-Hb results. Student $t$ tests were conducted to compare the mean differences in oxy-Hb between a rest and a task phase during the pre-switch session, the first conflict block in the post-switch session, and the redundant block in the post-switch session. Multiple comparisons were conducted; thus, we applied a strict $0.0125(0.05 / 4)$ alpha level of significance to the analyses (channel 4, 5, 13, 14).

In the pre-switch session, the results revealed significant activations in the right lateral prefrontal regions (channel 4,5 ) during the task phases compared to the rest phases (channel 4: $t$ $(35)=3.52, p<.001, d=0.84$; channel 5: $t(44)=3.63, p<.001, d=0.89)$. The left lateral prefrontal regions (channel 13,14) did not significantly activate during the task phases. In terms of the first conflict block in the post-switch session, Figure 4 shows how the same pattern of the greater activation in the right lateral prefrontal regions was observed (channel 4: $t(34)=3.77, p$ $<.001, d=0.87$; channel $5: t(41)=4.29, p<.001, d=1.00)$, whereas the left lateral prefrontal activation was close to a marginally significant level in one channel (channel 13:t $t(27)=2.35, p$ $=.026, d=0.49)$ and was not significant in another channel (channel 14: $t(42)=1.22, p=.228, d$ $=0.27$ ). In contrast, in the redundant block children did not exhibit any significant activation in 
the lateral prefrontal regions during the task phases (channel 4: $t(33)=1.34, p=.190, d=0.31$; channel 5: $t(39)=1.66, p=.106, d=0.40$; channel 13: $t(27)=-0.94, p=.354, d=0.25$; Note that channel 14 was close to a marginal significant level: $t(42)=2.27, p=.028, d=0.42$ ).

(Please insert Figure 4 about here)

\subsection{The relation between $f N I R S$ results and behavioral results in the revised goal neglect DCCS}

Based on the observed behavioral and neural data, we examined whether the activation in prefrontal regions during the redundant block would predict performance in the second conflict block of the post-switch session. To find this, we conducted mixed-effect regression analyses having the performance of the second conflict block as a dependent variable. As possible predictors, the following fixed-effect variables were included in the analysis: an age factor and factors of mean changes in oxy-Hb (channel $4,5,13,14)$ during the redundant block. The positive estimates of the mean changes in oxy- $\mathrm{Hb}$ indicated that increasing the average changes in oxy-Hb during the task phases as compared with the rest phases led to the better sorting performance in the second conflict block. A random intercept for participants was also included as a random effect variable. We further applied a strict $0.0125(0.05 / 4)$ alpha level of significance to the analyses (channel $4,5,13,14$ ). The analyses revealed no channels in which prefrontal activation predicted the performance on sorts of the second conflict block (see Table 4; Note that channel 4 showed a marginally significant effect). ${ }^{3}$ In addition, we conducted analyses including age-related interactions, showing no significant age-related interactions (see Appendix B). 
Table 4

Results of regression analysis examining the effects of prefrontal activation in each channel on the performance of the revised goal neglect DCCS

Fixed effect Estimate

(Channel 4)

$\begin{array}{llccc}\text { (Intercept) } & 0.805 & 0.039 & 20.70 & <.001 \\ \text { Age in months } & 0.014 & 0.004 & 3.26 & .003 \\ \text { Ch4_changes } & 0.614 & 0.260 & 2.35 & .021\end{array}$

(Channel 5)

$\begin{array}{ccccc}\text { (Intercept) } & 0.811 & 0.039 & 20.92 & <.001 \\ \text { Age in months } & 0.013 & 0.004 & 2.97 & .005 \\ \text { Ch5_changes } & 0.363 & 0.198 & 1.83 & .070\end{array}$

(Channel 13)

$\begin{array}{ccccc}\text { (Intercept) } & 0.863 & 0.040 & 21.77 & <.001 \\ \text { Age in months } & 0.011 & 0.005 & 2.05 & .049 \\ \text { Ch13_changes } & 0.307 & 0.416 & 0.74 & .463\end{array}$

(Channel 14)

$\begin{array}{ccccc}\text { (Intercept) } & 0.821 & 0.035 & 23.69 & <.001 \\ \text { Age in months } & 0.012 & 0.004 & 3.03 & .005 \\ \text { Ch14_changes } & 0.284 & 0.359 & 0.79 & .430\end{array}$

We returned to the raw data and noticed that lateral prefrontal activation during the redundant block had strengthened from the second cycle (Figure 5). One potential reason for the finding was that the children could not predict the presence of the upcoming phase of sorting 
conflict cards just after sorting consecutive redundant cards in the first cycle, but the experience of the first cycle would notify them of the necessity of holding goal representations in an active state for the upcoming phase throughout the second and third cycles. However, the preregistered analysis did not address effects of the number of cycles on the dependent variable. Therefore, we conducted mixed-effect regression analyses for exploration, in which an age factor, factors of mean changes in oxy- $\mathrm{Hb}$ (channel $4,5,13,14)$, factors regarding the number of cycles (first cycle $=-1$, second cycle $=0$. third cycle $=1$ ), and the interaction between number of cycles and the mean changes in oxy-Hb were included as fixed effect variables. A random intercept for the participant was also included as a random effect variable. As shown in Table 5, the exploratory analyses showed a significant main effect of the mean changes in oxy-Hb (channel 4) on the performance of the second conflict block, which indicated that the greater activation of the right lateral prefrontal region (channel 4) during the task phase of the redundant block could partially account for sorting conflict cards subsequently. We did not observe any significant main effects of the mean changes in oxy-Hb in other channels.

(Please insert Figure 5 about here) 
Table 5

Results of regression analysis examining the effects of prefrontal activation on channel 4 during the redundant block and cycles on the performance of the revised goal neglect DCCS

\begin{tabular}{ccccc}
\hline Fixed effect & Estimate & $S E$ & $t$ value & $\operatorname{Pr}(>|t|)$ \\
\hline (Intercept) & 0.802 & 0.040 & 20.08 & $<.001$ \\
Age in months & 0.014 & 0.004 & 3.30 & .003 \\
Cycle & 0.034 & 0.033 & 1.05 & .299 \\
Ch4_changes & 0.786 & 0.279 & 2.82 & .006 \\
Cycle $\times$ Ch4_changes & 0.667 & 0.380 & 1.76 & .084 \\
\hline
\end{tabular}

In addition to the preregistered plan, we conducted analyses for prefrontal activation during the second conflict block in the post-switch session. Although these analyses were motivated by the expectation that prefrontal activation during the second conflict block would be directly connected with goal neglect, our experiment design did not include a rest phase to contrast with a task phase during the second conflict block. This is because there was no break between the redundant and the second conflict block to examine whether prefrontal activation during the redundant block predicted the degree to which children neglected goal representations immediately after sorting consecutive redundant cards. Therefore, we tentatively compared the mean differences in oxy-Hb between a rest phase before the redundant block and a task phase during the second conflict block. Student $t$ tests revealed that children did not exhibit any significant activation in the lateral prefrontal regions during the second conflict block (see Appendix C). We also examined the relation between behavioral and neural results in the second conflict block, indicating that the interaction between the right prefrontal activation (channel 5) 
and a factor of cycles partially accounts for the performance of the second conflict block (discussed in Appendix C).

\section{Discussion}

The current study was designed to investigate the cognitive and neural underpinnings of goal maintenance. Thus far, no developmental study has addressed the neural mechanisms underlying active maintenance of goal representations, although neuroimaging studies of adults have shown that goal maintenance is linked with the activation of dorsolateral regions of the prefrontal cortex (e.g., Braver \& Cohen, 2001; MacDonald et al., 2000). Therefore, we not only attempted to replicate behavioral findings from the experimental paradigm inducing goal neglect (Marcovitch et al., 2010), but also to measure brain activation underlying goal maintenance in children with the revised paradigm. Our study yielded four main findings. First, it was demonstrated that children had difficulty with sorting conflict cards of the mostly redundant deck, replicating the findings from Marcovitch et al. (2007). Second, individual differences in inhibitory control uniquely predicted the performance on the mostly redundant deck, controlling for the developmental differences in working memory capacity. Third, children showed stronger activations in the right prefrontal regions when they sorted conflict cards, which is a replication of the findings from Moriguchi and Hiraki (2009, 2011). However, children did not exhibit significant prefrontal activations during redundant sorting when collapsing the three cycles. Fourth, taking changes of prefrontal activation across cycles into consideration, increased activation in at least one localized region of the right lateral prefrontal cortex during redundant sorting predicted the performance of the subsequent conflict sort.

\subsection{Cognitive underpinning of goal maintenance}


We succeeded in our aim to replicate the findings of the goal neglect DCCS in Marcovitch et al. (2007) $)^{4}$, indicating that young children have difficulty maintaining goal representations required especially in the mostly redundant deck. Given that participants in Marcovitch et al. (2007) are American children, our findings with Japanese children also imply that the phenomenon and cognitive underpinning of goal neglect can be observed across cultures, suggesting that goal maintenance behavior and processes may be universal. In line with this pattern, there are cross-cultural studies that have shown similarities in the development of executive functions among different cultures (e.g., Moriguchi, Evans, Hiraki, Itakura, \& Lee, 2012; Senzaki, Wiebe, Masuda, Shimizu, 2018). Directly examining cross-cultural comparisons of goal maintenance among young children is beyond our scope, but future studies can further inform this issue.

On the basis of the model of executive functions (e.g., Miyake \& Friedman, 2012) and working memory (Kane \& Engle, 2004), we considered that working memory tasks may potentially capture inhibitory control as well as working memory capacity. Thus, we focused on both working memory and inhibitory control as potential factors predicting goal maintenance and examined how each factor uniquely accounted for the sorting performance of the goal neglect DCCS. Our data highlighted relations of inhibitory control to the task performance, especially for the mostly redundant deck, indicating that inhibitory control is associated with goal maintenance required in the goal neglect DCCS. The findings were consistent with those from previous developmental research on shifting (Chevalier et al., 2012) and other paradigms for measuring goal neglect (Towse et al., 2007). Marcovitch et al. (2010) demonstrated age-related changes in the interaction between working memory capacity and the deck type, indicating that working memory capacity predicted the performance on the mostly redundant deck for both 4- 
year-olds and 6-year-olds, but it predicted the performance on the mostly redundant deck only for 4-year-olds. ${ }^{5}$ We could perfectly replicate this finding using same analysis as Marcovitch et al. (2010). These findings indicated, as suggested by Marcovitch et al. (2010), that the development of working memory capacity was a source of age-related effects on the goal neglect DCCS. However, an additional analysis including inhibitory control revealed no such age-related interactions with working memory capacity and inhibitory control (see Appendix A). Our data indicated that the predictive power of inhibitory control is so strong throughout early childhood that it could wipe out the age-related effect and account for the sorting performance beyond age. Furthermore, Marcovitch et al. (2010) found that sorting conflict cards in the mostly redundant deck and the mostly conflict deck are related but differentially rely on the two regulatory processes, namely, goal maintenance and conflict resolution. Our findings fit with their view. Even though inhibitory control was controlled, a trend of significant main effect of working memory capacity remained, suggesting that sorting the two types of deck might share common regulatory processes supported by working memory, possibly goal maintenance and conflict resolution. The interaction between inhibitory control and deck type reflects that sorting the two types of deck can be separable in the view of goal maintenance. These findings suggest one possibility that both the development of inhibitory control and working memory capacity potentially support maintaining goal representations in young children (but we discussed another possibility in the section of Limitation and Conclusion that inhibitory control is supported by goal maintenance). ${ }^{6}$

The influences of inhibitory control on goal maintenance can potentially stem from two independent sources (Friedman \& Miyake, 2004). In the DCCS, children needed to change their sorting behavior from using one rule to using another rule and resolve the within-stimulus 
conflict between the previous dimension and the new dimension. Therefore, first, inhibitory control may play a role in overcoming the previously relevant rule to represent the new one in working memory. Second, inhibitory control may contribute to the resistance to distracters in ignoring the stimulus feature associated with the irrelevant dimension. In this study, (1) the red/blue task mainly required children to inhibit the previously relevant rule and (2) the flanker task mainly required them to ignore distractors. It was possible that the two inhibitory tasks were differently associated with the two types of sorts in the original goal neglect DCCS. Our additional analysis, in which factors of the red/blue task and the fish flanker task were separately entered into the model, suggests that the red/blue task was closely related to both types of sorts in the original goal neglect DCCS possibly because both sorting performance require children to suppress the previously relevant rule to activate the newly relevant rule. In contrast, it was also shown that the fish flanker task was not related to both deck types of the original goal neglect DCCS, but it tended to be relatively sensitive to the differences of the sorting type. Given the results of post-hoc analyses, one possibility is that children with low fish flanker performance might pay more attention to the goal-irrelevant dimension of redundant cards in the original goal neglect DCCS and are more likely to lose sight of their goals. Thus, it can be expected that each inhibitory task is uniquely related to the different aspect of the original goal neglect DCCS. However, it is difficult for us to precisely discuss the contributions of these two potential inhibitory processes to goal maintenance, as our study was not constructed to examine this issue. For example, although we employed one task for measuring each of the two forms of inhibitory control, performance on each task also reflects nonexecutive processes due to the task impurity problem (Miyake et al., 2000). Using a confirmatory factor analysis and extracting common variance of several tasks measuring two forms of inhibitory control is an ideal way to handle this 
problem. In line with this consideration, Gandolfi, Viterbori, Traverso, and Usai (2014)

distinguished the two forms of inhibitory control in preschoolers. Furthermore, the task impurity problem was also true of measuring working memory capacity. Thus, future studies should use much purer measures of working memory and two forms of inhibitory control through the aforementioned approach and reexamine cognitive underpinning of goal maintenance.

\subsection{Neural underpinning of goal maintenance}

We were successful in replicating the findings from Moriguchi and Hiraki $(2009,2011)$, in which lateral prefrontal regions activate strongly while sorting conflict cards successfully in both the pre-switch session and the post-switch session. In the current study, we observed a laterality effect as well, that is, right lateral prefrontal regions significantly activated and left lateral prefrontal regions did not activate (but one channel in the left prefrontal regions was close to be marginally significant). However, contrary to our prediction, we could not observe such salient neural patterns while sorting redundant cards when collapsing three cycles and their associations with behavioral performance of the revised goal neglect DCCS. The behavioral performance of the revised goal neglect DCCS was modestly correlated with the performance on the mostly redundant deck in the original goal neglect DCCS $(r=0.49, p<.001)$, and inhibitory control could account for both task performance. Thus, in comparison to the original goal neglect DCCS, the validity of the revised goal neglect DCCS was guaranteed to some degree, so the unexpected results of neural patterns while sorting redundant cards might be explained by other factors than the insensitivity of the task as a behavioral measure. Indeed, reflecting over the effect of cycles allows us to examine the significant influence of the activation of right lateral prefrontal regions on the subsequent sorting behavior. Despite this analysis not being preregistered, it is the first evidence for the neural underpinning of goal maintenance in preschoolers, which is theoretically 
consistent with previous developmental research highlighting goal maintenance (e.g., Marcovitch et al., 2007, 2010). Marcovitch et al. (2007) also demonstrated that children fail to use the rules despite being told the rules immediately before every trial, which is a main tenet of goal neglect. Given that the revised goal neglect DCCS is closely related to the original goal neglect DCCS, it is expected that our findings from fNIRS would be replicated even if the rules were provided in every trial. To establish the link between the right prefrontal activations and goal maintenance, future studies should attempt to replicate our findings under the condition that children are told the rules every trial.

In term of the neural activity during the redundant block, there might be differences in the pattern of the lateral prefrontal activation between in the first cycle and in the second and third cycles. Our findings suggest that children did not activate the lateral prefrontal regions during the redundant block in the first cycle (see Figure 5). It is only after the experience of the first cycle that the activation level of right lateral prefrontal regions predicts sorting performance in the second conflict block. Thus, we needed to reflect over the effect of cycles to examine whether the prefrontal activation had impacts on the subsequent sorting performance. The effect of cycles possibly can be interpreted from the Dual Mechanism of Control framework that proposes that cognitive control operates via two distinct operating modes, that is, proactive control and reactive control (Braver, 2012; Braver et al., 2007). It has been demonstrated that young children, particularly children younger than five, tend to rely on reactive control (e.g., Chatham et al., 2009; Gonthier et al., 2019), and 5-year-olds were capable of engaging in proactive control only when reactive control was made more difficult (Chevalier, Martis, Curran, \& Munakata, 2015). In the current study, in the first cycle, children might not have tried to maintain task goals actively during the redundant block and might have resolved conflict information according to 
post-switch rules reactively in response to the unpredictable appearance of the conflict sort in the second conflict block. This is because when sorting the redundant cards during the redundant block in the fires cycle, they did not know the later requirement of the conflict card sorts. However, after recognizing that sorting conflict cards after sorting consecutive redundant cards is necessary, they might make efforts to proactively prepare for the subsequent conflict card sort during the redundant card sort. Therefore, one can infer that our participants would activate goal representations reactively in the first cycle, but in the second and third cycle they proactively maintain goal representations even during the redundant block. Given that adults mainly engage in proactive control, the results obtained from the second and third cycle would fit with the findings of adult neuroimaging studies (Braver \& Cohen, 2001; MacDonald et al., 2000).

Our findings provide additional evidence about the role of the right lateral prefrontal regions. The right lateral prefrontal regions were activated when children sorted conflict cards during the pre-switch session and the first conflict block as well as when children who were successful in the second conflict block sorted redundant cards. Thus, we suggest that the sustained activation of the right lateral prefrontal regions is closely related to goal maintenance and conflict resolution, which are required to correctly perform the DCCS. Moriguchi and Sakata (2020) reported results that are consistent with this pattern. They asked children to perform a social DCCS where those children sorted cards according to one dimension (execution phase) after observing another person sorting cards according to another dimension (observation phase), and they found that 4- to 6-year-olds exhibited lateral prefrontal activations during both phases. These findings suggest that lateral prefrontal regions may associate with some regulatory processes in the DCCS, that is, selecting relevant and ignoring irrelevant dimensions as well as maintaining a currently relevant task goal. 
Summing up the evidence, we can conclude that preschoolers recruit the right lateral prefrontal areas during successful conflict sorts (reflecting conflict resolution and goal maintenance) and redundant sorts with subsequent successful conflict sorts (reflecting goal maintenance).

\subsection{Limitations and conclusions}

Although our study generated significant findings, it was subject to some limitations. First, measures of working memory capacity and inhibitory control should be more valid and reliable to capture the two psychological constructs accurately. In terms of the reliability, our inhibition tasks, especially for the red/blue task, do not have a good internal consistency. One of reasons for the inaccurate reliability is the low number of trials, although we decided the number of trials according to the procedure of previous studies (Simpson \& Riggs, 2005; Zelazo et al., 2013). Thus, future studies need to perform more trials/sessions not to demand children too much or conduct the same structured task using different materials (red/blue task and grass/snow task, see Carlson, 2005). Furthermore, in terms of working memory, we conducted the backward digit span task. However, some researchers argue that the backward digit span task is more correlated with other short-term memory tasks than other working memory tasks, suggesting that the backward digit span task might not capture working memory capacity beyond short-term memory (e.g., Conway et al., 2005). Thus, in a future study we should perform more valid working memory tasks, such as a listening span test (Alloway, Gathercole, \& Pickering, 2006) and a color-naming span task (Camos \& Barrouillet, 2011). Second, we could not specify how inhibitory control interacted with goal maintenance. Although we assume that inhibitory control is one of factors underlying goal maintenance, there is a possibly that inhibitory control is supported by goal maintenance. For adults, Friedman et al. (2008) demonstrated that once the 
correlations with the common executive function factor were removed, there was no unique variance left for inhibition tasks, although shifting and updating tasks captured updating-specific and shifting-specific factors. They suggested that maintaining goal representations reflects a factor behind inhibition tasks more clearly than shifting and updating (Miyake \& Friedman, 2012). Recent developmental findings have also pointed to the key role of goal maintenance in the performance of inhibitory control tasks during early childhood (Blaye \& Chevalier, 2011; Barker \& Munakata, 2015; Lorsbach \& Reimers, 2010). In fact, there are theoretical positions that regard inhibitory control as a "by-product of goal maintenance" (see, Herd et al., 2014). Therefore, our current results can be interpreted in another way: that goal maintenance required in the goal neglect DCCS contributes to high performance on the inhibitory control tasks. Furthermore, we can also reconsider the role of the right prefrontal activation. We discussed that the sustained activation of the right lateral prefrontal regions is closely related to goal maintenance and conflict resolution, but the prefrontal activation might be mainly responsible for maintaining goal representations. According to another view (e.g., Herd et al., 2014), the strength of goal maintenance determines exerting conflict resolution. Thus, goal maintenance would be a source of sorting conflict cards during the pre-switch session and the first conflict block. The right prefrontal activation during the pre-switch session and the first conflict block can be interpreted as reflecting goal maintenance, like the right prefrontal activation during the redundant block. Therefore, future studies should investigate the causal relations between inhibitory control and goal maintenance in preschoolers. Third, although we discussed our results from the viewpoint of cognitive strategies, namely proactive/reactive control, we had no way to distinguish whether children engaged in proactive control or reactive control in the goal neglect DCCS. Future studies should directly address the neural underpinnings of goal maintenance 
using another paradigm for measuring proactive/reactive control such as child-friendly AX-CPT (Chatham et al., 2009; Lucenet \& Blaye, 2014) or a cue-based task switching paradigm

(Chevalier et al., 2015). Forth, the current study measured only prefrontal activation, but recent studies have demonstrated the importance of interactions among frontal and posterior brain regions on the development of executive functions (Buss \& Spencer, 2014, 2018). For instance, the activation in the prefrontal regions may be supported by the activation in the posterior regions in a bottom-up manner or may have an impact on the activation in the posterior regions in a top-down manner. These studies suggest that simply observing the prefrontal activation is not enough to address the neural mechanisms of how the prefrontal activation was activated. Thus, future studies should highlight the long-range connections between frontal and posterior regions.

Despite these potential limitations, the current findings provide the first evidence for the cognitive and neural underpinning of goal maintenance in young children. We have demonstrated that inhibitory control is closely related to the active maintenance of goal representations in preschoolers. In addition, we demonstrated that preschoolers start to recruit right lateral prefrontal areas not only while they resolve conflicting information but also while they maintain goal representations. We believe that our findings therefore offer important insights into the cognitive and neural underpinnings of goal maintenance and its roles in the control of goal-directed behavior. 


\section{Footnotes}

1. According to our initial calculation by $\mathrm{G}^{*}$ Power, this sample size was supposed to have $95 \%$ power to detect the effect size observed in Marcovitch et al. (2010). We preregistered our data collection plan based on this estimation and collected data from 61 children. It has later become apparent that the final sample size we used for analyses achieves around $71 \%$ power to detect the effect size. This was due to our miscalculation. We are indebted to a reviewer who suggested this correction.

2. After conducting the main analyses reported here, we confirmed that we could find similar findings even when we did not exclude the cycles where children could not sort perfectly in the first conflict block. The data exclusion was found to have little effect on our findings, but it was theoretically consistent with our key aim.

3. Without applying a strict alpha level of significance to analyses, the right prefrontal activation (Ch4) would be related to the performance on sorts of the second conflict block. However, we conducted multiple analyses due to lack of any predictions about a specific channel or a laterality effect, leading to more Type I errors. Thus, we did not interpret significant but uncorrected results.

4. Without a factor of inhibitory control tasks, our data also exhibited a significant main effect of working memory capacity and no significant interaction between working memory capacity and deck type, replicating the findings from Marcovitch et al. (2010).

5. Following the analysis procedure of Marcovitch et al. (2010), separate regression analyses were conducted on the proportion of correctly sorted conflict cards in the mostly redundant deck and that in the mostly conflict deck. These regression models were aimed to examine the interaction between working memory capacity and age in months. We found that the age- 
related interaction was significant in the mostly conflict deck $(b=-0.004, S E=0.002, t=$ 2.40, $p=.020)$, but not significant in the mostly redundant deck $(b=-0.004, S E=0.003, t=-$ $1.42, p=.162$ ), showing the successful replication of the findings from Marcovitch et al. (2010).

6. Given that working memory and inhibitory control may have a unique effect on the goal neglect DCCS, it is possible that executive functions in preschoolers would be composed of related but separable processes or factors, rather than a unitary factor. The current study is not designed to strongly support that the two-factor model fits the data for preschoolers better than the unitary factor model, but our findings should give us a clue that leads to clarification of the structure of executive functions in preschoolers. 


\section{Appendix A}

Age-related interactions with behavioral performance in the original and revised goal neglect $D C C S$

In terms of the performance of the original goal neglect DCCS, we conducted regression analysis including age-related interactions with standardized working memory capacity, inhibitory control, and deck type. As shown in Table A.1, there were not any significant agerelated interactions with the individual difference factors and the deck types. Importantly, the interaction between inhibitory control and deck type remained significant even though agerelated developmental trends were taken into consideration.

Next, the mixed model including age-related interactions with working memory capacity and inhibitory control was also tested with the performance on sorts in the second conflict block as a dependent variable. We found that age-related interactions were not significant and a main effect of inhibitory control was reduced to be not significant (see Table A.2). This finding suggests that the main effect of inhibitory control might be wiped out by age-related interactions, but adding the age-related interactions did not improve model fit $\left(\chi^{2}=1.16, d f=2, p=.560\right)$.

These findings suggest that working memory capacity and inhibitory control partially account for the effects of age on the performance of the original goal neglect DCCS, but their effects do not depend on age in months. From the viewpoint of parsimonious models, the model without age-related interactions with working memory capacity and inhibitory control fitted better with our sample. 
Table A.1

Results of regression analysis examining the effects of working memory capacity, inhibitory control, deck type, and age-related interactions on the performance of the original goal neglect DCCS

\begin{tabular}{|c|c|c|c|c|}
\hline Fixed effect & Estimate & $S E$ & $t$ value & $\operatorname{Pr}(>|t|)$ \\
\hline (Intercept) & 0.799 & 0.028 & 28.99 & $<.001$ \\
\hline Age in months & -0.002 & 0.004 & -0.38 & .705 \\
\hline Order (dimension) & 0.015 & 0.024 & 0.64 & .521 \\
\hline Order (condition) & -0.006 & 0.023 & -0.26 & .795 \\
\hline Deck type & 0.148 & 0.028 & 5.36 & $<.001$ \\
\hline Age $\times$ Deck type & 0.001 & 0.004 & 0.17 & .864 \\
\hline WM_composite & 0.036 & 0.019 & 1.90 & .060 \\
\hline Inhibit_composite & 0.071 & 0.025 & 2.88 & .005 \\
\hline Deck type $\times$ WM_composite & 0.004 & 0.019 & 0.22 & .825 \\
\hline Age $\times$ WM_composite & -0.001 & 0.002 & -0.59 & .554 \\
\hline Deck type $\times$ Inhibit_composite & -0.057 & 0.024 & -2.35 & .020 \\
\hline Age $\times$ Inhibit_composite & -0.0002 & 0.003 & -0.11 & .914 \\
\hline Age $\times$ Deck type $\times$ WM_composite & -0.002 & 0.003 & -0.71 & .480 \\
\hline Age $\times$ Deck type $\times$ Inhibit_composite & -0.001 & 0.002 & -0.36 & .723 \\
\hline
\end{tabular}


Table A. 2

Results of regression analysis examining the effects of working memory capacity, inhibitory control, and age-related interactions on the performance of the revised goal neglect DCCS

\begin{tabular}{ccccc}
\hline Fixed effect & Estimate & $S E$ & $t$ value & $\operatorname{Pr}(>|t|)$ \\
\hline (Intercept) & 0.843 & 0.033 & 25.78 & $<.001$ \\
Age in months & 0.006 & 0.004 & 1.28 & .209 \\
Order (dimension) & 0.018 & 0.028 & 0.64 & .526 \\
WM_composite & 0.043 & 0.022 & 1.97 & .055 \\
Inhibit_composite & 0.038 & 0.029 & 1.18 & .244 \\
Age $\times$ WM_composite & -0.002 & 0.003 & -0.69 & .496 \\
Age $\times$ Inhibit_composite & -0.001 & 0.003 & -0.28 & .780 \\
\hline
\end{tabular}




\section{Appendix B}

Age-related interactions with prefrontal activation

We examined age-related changes in the relations between the activation in prefrontal regions during the redundant block and performance in the second conflict block of the postswitch session. The mixed models including an age factor, factors of mean changes in oxy- $\mathrm{Hb}$ (channel $4,5,13,14$ ) during the redundant block, and their interactions were tested. A random effect variable was a random intercept for participant. Results showed no significant age-related interactions and no channels in which prefrontal activation predicted sorting performance in the second conflict block (see Table B.1). Thus, prefrontal activation during the redundant block did not have any effects on the performance in the second switch block, and the effects did not depend on age in months.

Table B.1

Results of regression analysis examining the effects of prefrontal activation in each channel on the performance of the revised goal neglect DCCS

Fixed effect $\quad$ Estimate $S E \quad t$ value $\operatorname{Pr}(>|t|)$

(Channel 4)

$\begin{array}{ccccc}\text { (Intercept) } & 0.812 & 0.038 & 20.89 & <.001 \\ \text { Age in months } & 0.015 & 0.004 & 3.42 & .002 \\ \text { Ch4_changes } & 0.627 & 0.261 & 2.40 & .019 \\ \text { Age } \times \text { Ch4_changes } & -0.032 & 0.028 & -1.16 & .251\end{array}$

(Channel 5)

$\begin{array}{ccccc}\text { (Intercept) } & 0.812 & 0.039 & 20.85 & <.001 \\ \text { Age in months } & 0.014 & 0.004 & 3.12 & .004 \\ \text { Ch5_changes } & 0.290 & 0.206 & 1.40 & .162 \\ \text { Age } \times \text { Ch5_changes } & -0.031 & 0.024 & -1.30 & .196\end{array}$


(Channel 13)

$\begin{array}{ccccc}\text { (Intercept) } & 0.859 & 0.040 & 21.05 & <.001 \\ \text { Age in months } & 0.010 & 0.005 & 1.90 & .067 \\ \text { Ch13_changes } & 0.300 & 0.419 & 0.72 & .476 \\ \text { Age } \times \text { Ch13_changes } & -0.022 & 0.046 & -0.48 & .637\end{array}$

(Channel 14)

\begin{tabular}{ccccc} 
(Intercept) & 0.821 & 0.035 & 23.59 & $<.001$ \\
Age in months & 0.012 & 0.004 & 3.06 & .004 \\
Ch14_changes & 0.272 & 0.361 & 0.75 & .453 \\
Age $\times$ Ch14_changes & -0.025 & 0.048 & -0.52 & .601 \\
\hline
\end{tabular}




\section{Appendix C}

\section{Prefrontal activation during the second conflict block}

In addition to the preregistered analyses plan, we tested whether lateral prefrontal regions would activate while sorting conflict cards in the second conflict block. Although children are likely to neglect goal representations in the second conflict block due to the adjacent redundant block, children who are successful in sorting conflict cards in this block would recruit lateral prefrontal regions, especially for right lateral prefrontal regions, based on the results of the first conflict block. All of the children finished sorting six conflict cards within 15 seconds, thus we assumed 15 seconds just after the redundant block to be a task phase during the second conflict block and calculated temporal changes in oxy- $\mathrm{Hb}$. As mentioned in the main text, we tentatively compared the mean differences in oxy-Hb between the rest phase before the redundant block and the task phase during the second conflict block. Student $t$ tests revealed that children did not exhibit any significant activation in the lateral prefrontal regions during the second conflict block $($ channel 4: $t(34)=-0.62, p=.543, d=0.18$; channel 5: $t(38)=-0.51, p=.615, d=0.21$; channel 13: $t(27)=-1.98, p=.058, d=0.49$; channel 14: $t(42)=0.22, p=.824, d=0.06)$. These results are similar to findings from the redundant block.

We also examined whether lateral prefrontal activation during the second conflict block was associated with the sorting performance in the same block. In reference to our results about the relations between the prefrontal activation during the redundant block and the performance in the second conflict block, a main effect of cycles and their interaction should be taken into consideration. Thus, we conducted mixed-effect regression analyses, in which an age factor, factors of mean changes in oxy-Hb (channel 4, 5, 13, 14), factors regarding the number of cycles, and the interaction between number of cycles and the mean changes in oxy-Hb were included as 
fixed effect variables, and a random intercept for participant was also included as a random effect variable. As a result, a main effect of prefrontal activation on each channel was not significant, but it is noteworthy that the interaction between the right prefrontal activation (channel 5) and a factor of cycles was significant (see Table C.1). According to the post-hoc analysis, during the earlier cycle the greater activation of the right lateral prefrontal region (channel 5) was negatively related to sorting conflict cards in the second conflict block ( $b=$ $1.27, t=-2.30, p=.024)$, but in the later cycle the greater right prefrontal activation was positively related, although the effect was not statistically significant $(b=0.50, t=1.49, p$ $=.141)$.

We interpreted these findings from both a methodological limited viewpoint and a theoretical viewpoint. First, methodological problems potentially cause no main effect on the prefrontal activation. Given the standard procedure where the duration time of a task phase was 20 seconds and children sorted about 8 conflict cards (Moriguchi \& Hiraki, 2009), the number of trials ( 6 conflict cards) and relatively short duration time ( 15 seconds) were not sufficient to capture gradual increases in oxy-Hb accurately. Indeed, some of children who were successful in all of the trials finished the second conflict block within 10 seconds, and it is likely that our results cannot reflect their increased prefrontal activation accurately. Second, the interaction with the number of cycles can be considered from the viewpoint of error detection and correction. In the earlier cycle, most children could not expect that they needed to sort conflict cards after sorting consecutive redundant cards, thus some of them made mistakes of sorting conflict cards due to goal neglect. However, it is possible that those children detected their own errors, which signaled for the engagement of the lateral prefrontal cortex to increase cognitive control and optimize goal-directed behavior (Fitzgerald et al., 2013; Rueda, Posner, \& Rothbart, 2004). In 
line with this possibility, brain activation related to inhibitory control has so far been confirmed even during a small number of errors, and the brain activation was caused by different mechanisms from those concerning successful overcoming conflict information (e.g., Braver et al., 2001; Rubia, Smith, Brammer, \& Taylor, 2003). As a result, children who obtained lower scores might recruit lateral prefrontal activation in response to their own errors. On the other hand, in the later cycle they probably noticed the task structure and maintained goal representations from the beginning of the redundant block. Therefore, the number of participants who made mistakes due to goal neglect decreased and the greater right prefrontal activation during the second block came to be positively related with the sorting performance.

Even if the abovementioned possibilities can partially account for our results, this study was not designed to examine the lateral prefrontal activation during the second conflict block, thus we cannot draw a strong conclusion. However, it is important to note that these findings and findings from the redundant block have common points that a factor of cycles has influences on the relation between the activation of right lateral prefrontal regions and the sorting behavior in the second conflict block.

Table C.1

Results of regression analysis examining the effects of prefrontal activation on channel 5 during the second conflict block and cycles and cycles on the performance of the revised goal neglect DCCS

\begin{tabular}{ccccc} 
Fixed effect & Estimate & $S E$ & $t$ value & $\operatorname{Pr}(>|t|)$ \\
\hline (Intercept) & 0.804 & 0.037 & 21.65 & $<.001$ \\
Age in months & 0.015 & 0.004 & 3.47 & .001 \\
Cycle & 0.073 & 0.029 & 2.55 & .132 \\
Ch5_changes & -0.383 & 0.335 & -1.15 & .255 \\
Cycle $\times$ Ch5_changes & 1.081 & 0.381 & 2.84 & .006 \\
\hline
\end{tabular}




\section{Acknowledgements}

We are grateful to the staff and students of the kindergarten schools for their participation in this research. We further thank the reviewers for their valuable and insightful comments and suggestions.

\section{Funding}

The research reported here was supported by a Grant-in-Aid for Japan Society for the Promotion of Science (JSPS) fellows (16J0372). 


\section{References}

Alloway, T. P., Gathercole, S. E., \& Pickering, S. J. (2006). Verbal and visuospatial short-term and working memory in children: Are they separable?. Child Development, 77, 1698-1716. https://doi.org/10.1111/j.1467-8624.2006.00968.x

Barker, J. E., \& Munakata, Y. (2015). Time isn't of the essence: Activating goals rather than imposing delays improves inhibitory control in children. Psychological Science, 26, 18981908. https://doi.org/10.1177\%2F0956797615604625

Best, J. R., \& Miller, P. H. (2010). A developmental perspective on executive function. Child Development, 81, 1641-1660. doi: 10.1111/j.1467-8624.2010.01499.x

Bates, D., Maechler, M., Bolker, B., \& Walker, S. (2015). 1me4: Linear mixed-effects models using Eigen and S4. R package version 1.1-9. <https://CRAN.R-project. org/package=lme4>.

Blackwell, K. A., Cepeda, N. J., \& Munakata, Y. (2009). When simple things are meaningful: Working memory strength predicts children's cognitive flexibility. Journal of Experimental Child Psychology, 103, 241-249. doi: 10.1016/j.jecp.2009.01.002

Blaye, A., \& Chevalier, N. (2011). The role of goal representation in preschoolers' flexibility and inhibition. Journal of Experimental Child Psychology, 108, 469-483. http://dx.doi.org/10.1016/j.jecp.2010.09.006

Braver, T. S. (2012). The variable nature of cognitive control: A dual mechanisms framework. Trends in Cognitive Sciences, 16, 106-113. doi:10.1016/j.tics.2011.12.010

Braver, T. S., Barch, D. M., Gray, J. R., Molfese, D. L., \& Snyder, A. (2001). Anterior cingulate cortex and response conflict: effects of frequency, inhibition and errors. Cerebral Cortex, 11, 825-836. https://doi.org/10.1093/cercor/11.9.825 
Braver, T. S., \& Cohen, J. D. (2001). Working memory, cognitive control, and the prefrontal cortex: Computational and empirical studies. Cognitive Processing, 2, 25-55.

Braver, T. S., Gray, J. R., \& Burgess, G. C. (2007). Explaining the many varieties of working memory variation: Dual mechanisms of cognitive control. In A. R. A. Conway, C. Jarrold, M. J. Kane, A. Miyake, \& J. N. Towse (Eds.), Variation in working memory (pp. 76-106). Oxford, England: Oxford University Press.

Buss, A.T., \& Spencer, J.P. (2014). The emergent executive: A dynamic neural field theory of the development of executive function. Monographs of the Society for Research in Child Development, 79, 1-104. doi: 10.1002/mono.12096

Buss, A. T., \& Spencer, J. P. (2018). Changes in frontal and posterior cortical activity underlie the early emergence of executive function. Developmental Science, 21, e12602. https://doi.org/10.1111/desc.12602

Camos, V., \& Barrouillet, P. (2011). Developmental change in working memory strategies: From passive maintenance to active refreshing. Developmental Psychology, 47, 898904. https://doi.org/10.1037/a0023193

Carlson, S. M. (2005). Developmentally sensitive measures of executive function in preschool children. Developmental Neuropsychology, 28, 595-616. https://doi.org/10.1207/s15326942dn2802_3

Case, R., Kurland, M., \& Goldberg, J. (1982). Operational efficiency and the growth of shortterm memory span. Journal of Experimental Child Psychology, 33, 386-404. https://doi.org/10.1016/0022-0965(82)90054-6 
Chatham, C. H., Claus, E. D., Kim, A., Curran, T., Banich, M. T., \& Munakata, Y. (2012).

Cognitive control reflects context monitoring, not motoric stopping, in response inhibition. PloS one, 7, e31546.

Chatham, C. H., Frank, M. J., \& Munakata, Y. (2009). Pupillometric and behavioral markers of a developmental shift in the temporal dynamics of cognitive control. Proceedings of the National Academy of Sciences, 106, 5529-5533. doi:10.1073/pnas.0810002106

Chevalier, N., \& Blaye, A. (2008). Cognitive flexibility in preschoolers: the role of representation activation and maintenance. Developmental Science, 11, 339-353. doi: 10.1111/j.1467-7687.2008.00679.x.

Chevalier, N., Chatham, C. H., \& Munakata, Y. (2014). The practice of going helps children to stop: The importance of context monitoring in inhibitory control. Journal of Experimental Psychology: General, 143, 959-965. doi: 10.1037/a0035868.

Chevalier, N., Martis, S. B., Curran, T., \& Munakata, Y. (2015). Metacognitive processes in executive control development: The case of reactive and proactive control. Journal of Cognitive Neuroscience, 27, 1125-1136. doi:10.1162/jocn

Chevalier, N., Sheffield, T. D., Nelson, J. M., Clark, C. A., Wiebe, S. A., \& Espy, K. A. (2012). Underpinnings of the costs of flexibility in preschool children: the roles of inhibition and working memory. Developmental Neuropsychology, 37, 99-118. https://doi.org/10.1080/87565641.2011.632458

Conway, A. R., Kane, M. J., Bunting, M. F., Hambrick, D. Z., Wilhelm, O., \& Engle, R. W. (2005). Working memory span tasks: A methodological review and user's guide. Psychonomic Bulletin \& Review, 12, 769-786. https://doi.org/10.3758/BF03196772 
Diamond, A. (2013). Executive functions. Annual review of psychology, 64, 135-168. https://doi.org/10.1146/annurev-psych-113011-143750

Dreisbach, G., \& Goschke, T. (2004). How positive affect modulates cognitive control: reduced perseveration at the cost of increased distractibility. Journal of Experimental Psychology: Learning, Memory, and Cognition, 30, 343-353. http://dx.doi.org/10.1037/02787393.30 .2 .343

Duncan, J., Burgess, P., \& Emslie, H. (1995). Fluid intelligence after frontal lobe lesions. Neuropsychologia, 33, 261-268. https://doi.org/10.1016/0028-3932(94)00124-8

Duncan, J., Emslie, H., Williams, P., Johnson, R., \& Freer, C. (1996). Intelligence and the frontal lobe: The organization of goal-directed behavior. Cognitive Psychology, 30, 257-303. https://doi.org/10.1006/cogp.1996.0008

Engle, R. W., \& Kane, M. J. (2004). Executive Attention, Working Memory Capacity, and a Two-Factor Theory of Cognitive Control. In B. Ross (Ed.), The Psychology of Learning and Motivation, Vol. 44, (pp. 145-199). New York, NJ: Elsevier.

Firbank, M., Okada, E., \& Delpy, D. T. (1998). A theoretical study of the signal contribution of regions of the adult head to near-infrared spectroscopy studies of visual evoked responses. Neuroimage, 8, 69-78. doi: 10.1006/nimg.1998.0348

Fiske, A., \& Holmboe, K. (2019). Neural substrates of early executive function development. Developmental Review, 52, 42-62. https://doi.org/10.1016/j.dr.2019.100866

Fitzgerald, K. D., Liu, Y., Stern, E. R., Welsh, R. C., Hanna, G. L., Monk, C. S., ... \& Taylor, S. F. (2013). Reduced error-related activation of dorsolateral prefrontal cortex across pediatric anxiety disorders. Journal of the American Academy of Child \& Adolescent Psychiatry, 52, 1183-1191. https://doi.org/10.1016/j.jaac.2013.09.002 
Friedman, N. P., \& Miyake, A. (2004). The relations among inhibition and interference control functions: A latent variable analysis. Journal of Experimental Psychology: General, 133, 101-135. doi: 10.1037/0096-3445.133.1.101.

Friedman N. P., Miyake, A., Young, S. E., DeFries, J. C., Corley, R. P., \& Hewitt, J. K. (2008). Individual differences in executive functions are almost entirely genetic in origin. Journal of Experimental Psychology: General, 137, 201-225. doi: 10.1037/0096-3445.137.2.201.

Gandolfi, E., Viterbori, P., Traverso, L., \& Usai, M. C. (2014). Inhibitory processes in toddlers: a latent-variable approach. Frontiers in Psychology, 5, 381. https://doi.org/10.3389/fpsyg.2014.00381

Garon, N., Bryson, S. E., \& Smith, I. M. (2008). Executive function in preschoolers: A review using an integrative framework. Psychological Bulletin, 134, 31-60. doi: 10.1037/00332909.134.1.31.

Gonthier, C., Zira, M., Colé, P., \& Blaye, A. (2019). Evidencing the developmental shift from reactive to proactive control in early childhood and its relationship to working memory. Journal of Experimental Child Psychology, 177, 1-16. https://doi.org/10.1016/j.jecp.2018.07.001

Herd, S., O’Reilly, R., Hazy, T., Chatham, C., Brant, A., \& Friedman, N. (2014). A Neural network model of individual differences in task switching abilities. Neuropsychologia, 62, 375-389. https://doi.org/10.1016/j.neuropsychologia.2014.04.014

Herrmann, M. J., Ehlis, A. C., \& Fallgatter, A. J. (2003). Prefrontal activation through task requirements of emotional induction measured with NIRS. Biological Psychology, 64, 255263. https://doi.org/10.1016/S0301-0511(03)00095-4 
Hughes, C., Ensor, R., Wilson, A., \& Graham, A. (2010). Tracking executive function across the transition to school: A latent variable approach. Developmental Neuropsychology, 35, 20-36. http://dx.doi.org/ 10.1080/87565640903325691

Huppert, T. J., Diamond, S. G., Franceschini, M. A., \& Boas, D. A. (2009). HomER: A review of time-series analysis methods for near-infrared spectroscopy of the brain. Applied Optics, 48, D280-D298.

Kane, M. J., \& Engle, R. W. (2003). Working-memory capacity and the control of attention: the contributions of goal neglect, response competition, and task set to Stroop interference. Journal of Experimental Psychology: General, 132, 47-70. http://dx.doi.org/10.1037/0096-3445.132.1.47

Lerner, M. D., \& Lonigan, C. J. (2014). Executive function among preschool children: Unitary versus distinct abilities. Journal of Psychopathology and Behavioral Assessment, 36, 626639. http://dx.doi.org/10.1007/s10862-014-9424-3

Lorsbach, T. C., \& Reimer, J. F. (2010). Developmental differences in cognitive control: Goal representation and maintenance during a continuous performance task. Journal of Cognition and Development, 11, 185-216. https://doi.org/10.1080/15248371003699936

Lopez-Garcia, P., Lesh, T. A., Salo, T., Barch, D. M., MacDonald, A. W., Gold, J. M., ... \& Carter, C. S. (2016). The neural circuitry supporting goal maintenance during cognitive control: a comparison of expectancy AX-CPT and dot probe expectancy paradigms. Cognitive, Affective, \& Behavioral Neuroscience, 16, 164-175. doi: 10.3758/s13415-015-0384-1. 
Lucenet, J., \& Blaye, A. (2014). Age-related changes in the temporal dynamics of executive control: a study in 5- and 6-year-old children. Frontiers in Psychology, 5, 1-11. doi:10.3389/fpsyg.2014.00831

MacDonald III, A. W., \& Carter, C. S. (2003). Event-related FMRI study of context processing in dorsolateral prefrontal cortex of patients with schizophrenia. Journal of Abnormal Psychology, 112, 689. doi:10.1037/0021-843X.112.4.689

MacDonald, A. W., Cohen, J. D., Stenger, V. A., \& Carter, C. S. (2000). Dissociating the role of the dorsolateral prefrontal and anterior cingulate cortex in cognitive control. Science, 288, 1835-1838. doi:10.1126/science.288.5472.1835

Marcovitch, S., Boseovski, J. J., \& Knapp, R. J. (2007). Use it or lose it: Examining preschoolers' difficulty in maintaining and executing a goal. Developmental Science, 10, 559-564. doi:10.1111/j.1467-7687.2007.00611.x

Marcovitch, S., Boseovski, J. J., Knapp, R. J., \& Kane, M. J. (2010). Goal neglect and working memory capacity in 4-to 6-Year-Old Children. Child Development, 81, 1687-1695. http://dx.doi.org/10.1111/j.1467-8624.2010.01503.x

Marton, K., Kelmenson, L., \& Pinkhasova, M. (2007). Inhibition control and working memory capacity in children with SLI. Psychologia, 50, 110-121. https://doi.org/10.2117/psysoc.2007.110

McVay, J. C., \& Kane, M. J. (2009). Conducting the train of thought: working memory capacity, goal neglect, and mind wandering in an executive-control task. Journal of Experimental Psychology: Learning, Memory, and Cognition, 35, 196-204. doi: 10.1037/a0014104 Mehnert, J., Akhrif, A., Telkemeyer, S., Rossi, S., Schmitz, C. H., Steinbrink, J., . . Neufang, S. (2013). Developmental changes in brain activation and functional connectivity during 
response inhibition in the early childhood brain. Brain and Development, 35, 894-904. doi:10.1016/j.braindev.2012.11.006

Miller, E. K., \& Cohen, J. D. (2001). An integrative theory of prefrontal cortex function. Annual Review of Neuroscience, 24, 167-202. http://dx .doi.org/10.1146/annurev.neuro.24.1.167

Miller, M. R., Giesbrecht, G. F., Müller, U., McInerney, R. J., \& Kerns, K. A. (2012). A latent variable approach to determining the structure of executive function in preschool children. Journal of Cognition and Development, 13, 395-423.

http://dx.doi.org/10.1080/15248372.2011.585478

Miyake, A., \& Friedman, N. P. (2012). The nature and organization of individual differences in executive functions: four general conclusions. Current Directions in Psychological Science, 21, 8-14. doi: $10.1177 / 0963721411429458$

Miyake, A., Friedman, N. P., Emerson, M. J., Witzki, A. H., Howerter, A., \& Wager, T. D. (2000). The unity and diversity of executive functions and their contributions to complex "Frontal Lobe" tasks: a latent variable analysis. Cognitive Psychology, 41, 49-100. doi:10.1006/cogp.1999.0734

Moriguchi, Y., Evans, A. D., Hiraki, K., Itakura, S., \& Lee, K. (2012). Cultural differences in the development of cognitive shifting: East-West comparison. Journal of Experimental Child Psychology, 111(2), 156-163. https://doi.org/10.1016/j.jecp.2012.04.009

Moriguchi, Y., \& Hiraki, K. (2009). Neural origin of cognitive shifting in young children. Proceedings of the National Academy of Sciences of the United States of America, 106, 60176021. doi:10.1073/pnas.0809747106 
Moriguchi, Y., \& Hiraki, K. (2011). Longitudinal development of prefrontal function during early childhood. Developmental Cognitive Neuroscience, 1, 153-162. doi:10.1016/j.den.2010.12.004

Moriguchi, Y., \& Hiraki, K. (2013). Prefrontal cortex and executive function in young children: a review of NIRS studies. Frontiers in Human Neuroscience, 7, 867. http://dx.doi.org/10.3389/fnhum.2013.00867

Moriguchi, Y., \& Sakata, C. (2020). Development of Cognitive Shifting from Others' Behavior in Young Children: A Near-infrared Spectroscopy Study. Developmental Neuropsychology, 1-9. https://doi.org/10.1080/87565641.2019.1710512

Moriguchi, Y., \& Shinohara, I. (2018). Effect of the COMT Val158Met genotype on lateral prefrontal activations in young children. Developmental Science, e12649. doi:10.1111/desc. 12649

Morton, J. B., \& Munakata, Y. (2002). Active versus latent representations: A neural network model of perseveration, dissociation, and decalage. Developmental Psychobiology, 40, 255265. https://doi.org/10.1002/dev.10033

Munakata, Y., Snyder, H. R., \& Chatham, C. H. (2012). Developing cognitive control: three key transitions. Current Directions in Psychological Science, 21, 71-77. doi: $10.1177 / 0963721412436807$

Okamoto, M., Dan, H., Sakamoto, K., Takeo, K., Shimizu, K., Kohno, S., ... \& Dan, I. (2004). Three-dimensional probabilistic anatomical cranio-cerebral correlation via the international 10-20 system oriented for transcranial functional brain mapping. Neuroimage, 21, 99-111. https://doi.org/10.1016/j.neuroimage.2003.08.026 
Paxton, J. L., Barch, D. M., Racine, C. A., \& Braver, T. S. (2007). Cognitive control, goal maintenance, and prefrontal function in healthy aging. Cerebral Cortex, 18, 1010-1028. https://doi.org/10.1093/cercor/bhm135

Perlman, S. B., Huppert, T. J., \& Luna, B. (2016). Functional near-infrared spectroscopy evidence for development of prefrontal engagement in working memory in early through middle childhood. Cerebral Cortex, 26, 2790-2799. doi:10.1093/cercor/bhv139

R Core Team. (2013). R: A language and environment for statistical computing. Vienna, Austria: R Foundation for Statistical Computing.

Rubia, K., Smith, A. B., Brammer, M. J., \& Taylor, E. (2003). Right inferior prefrontal cortex mediates response inhibition while mesial prefrontal cortex is responsible for error detection. Neuroimage, 20, 351-358. https://doi.org/10.1016/S1053-8119(03)00275-1

Rueda, M. R., Fan, J., McCandliss, B. D., Halparin, J. D., Gruber, D. B., Lercari, L. P., et al. (2004). Development of attentional networks in childhood. Neuropsychologia, 42, 10291040. https://doi.org/10.1016/j.neuropsychologia.2003.12.012

Rueda, M. R., Posner, M. I., \& Rothbart, M. K. (2004). Attentional control and self-regulation. In R. F. Baumeister \& K. D. Vohs (Eds.), Handbook of self-regulation: Research, theory, and applications (pp. 283-300). New York: The Guilford Press.

Rubia, K., Smith, A. B., Brammer, M. J., \& Taylor, E. (2003). Right inferior prefrontal cortex mediates response inhibition while mesial prefrontal cortex is responsible for error detection. Neuroimage, 20, 351-358. https://doi.org/10.1016/S1053-8119(03)00275-1

Saager, R. B., Telleri, N. L., \& Berger, A. J. (2011). Two-detector corrected near infrared spectroscopy (C-NIRS) detects hemodynamic activation responses more robustly than singledetector NIRS. Neuroimage, 55, 1679-1685. doi:10.1016/j.neuroimage.2011.01.043 
Senzaki, S., Wiebe, S. A., Masuda, T., \& Shimizu, Y. (2018). A cross-cultural examination of selective attention in Canada and Japan: The role of social context. Cognitive Development, 48, 32-41. https://doi.org/10.1016/j.cogdev.2018.06.005

Simpson, A., \& Riggs, K. J. (2005). Inhibitory and working memory demands of the day-night task in children. British Journal of Developmental Psychology, 10, 1-17. http://dx.doi.org/10.1348/026151005X28712

Smith, E., Anderson, A., Thurm, A., Shaw, P., Maeda, M., Chowdhry, F., .. . Gandjbakhche, A. (2017). Prefrontal activation during executive tasks emerges over early childhood: Evidence from functional Near Infrared Spectroscopy. Developmental Neuropsychology, 42, 253-264. doi:10.1080/87565641.2017.1318391

Sumitani, S., Tanaka, T., Tayoshi, S. Y., Ota, K., Kameoka, N., Ueno, S. I., \& Ohmori, T. (2006). Activation of the prefrontal cortex during the wisconsin card sorting test as measured by multichannel near-infrared spectroscopy. Neuropsychobiology, 53, 70-76. https://doi.org/10.1159/000091722

Tsujimoto, S., Yamamoto, T., Kawaguchi, H., Koizumi, H., \& Sawaguchi, T. (2004). Prefrontal cortical activation associated with working memory in adults and preschool children: An event-related optical topography study. Cerebral Cortex, 14, 703-712. doi:10.1093/cercor/bhh030

Towse, J. N., Lewis, C., \& Knowles, M. (2007). When knowledge is not enough: the phenomenon of goal neglect in preschool children. Journal of Experimental Child Psychology, 96, 320-332. http://dx.doi.org/10.1016/j.jecp.2006.12.007 
Usai, M. C., Viterbori, P., Traverso, L., \& De Franchis, V. (2014). Latent structure of executive function in five-and six-year-old children: a longitudinal study. European Journal of Developmental Psychology, 11, 447-462. http://dx.doi.org/10.1080/17405629.2013.840578

Wiebe, S. A., Espy, K. A., \& Charak, D. (2008). Using confirmatory factor analysis to understand executive control in preschool children: I. Latent structure. Developmental Psychology, 44, 575-587. http://dx.doi.org/10.1037/0012-1649.44.2.575

Yamada, T., Umeyama, S., \& Matsuda, K. (2012). Separation of fNIRS signals into functional and systemic components based on differences in hemodynamic modalities. PLoS ONE, 7, e50271. https://doi.org/10.1371/journal.pone.0050271

Zelazo, P. D., Anderson, J. E., Richler, J., Wallner-Allen, K., Beaumont, J. L., \& Weintraub, S. (2013). II. NIH Toolbox Cognition Battery (CB): Measuring executive function and attention. Monographs of the Society for Research in Child Development, 78, 16-33.

Zelazo, P., Müller, U., Frye, D., \& Marcovitch, S. (2003). The development of executive function in early childhood. Monographs of the Society for Research in Child Development, $68,1-137$. 


\section{Figure captions}

Figure 1. The experimental sequence in the revised goal neglect DCCS

Figure 2. The fNIRS probe was attached to the lateral prefrontal region. Each channel consisted of one emitter optode and one detector optode. The regions of interest were located near F3/4, corresponding to Channels 2, 4, and 5, and Channels 11,13 , and 14, respectively

Figure 3. Proportion of sorting conflict cards correctly by age in months and deck type.

Figure 4. Temporal changes in the oxy- $\mathrm{Hb}$ (red) and deoxy- $\mathrm{Hb}$ (blue) concentration within the right lateral prefrontal areas (channel 4, 5) and the left lateral prefrontal areas (channel 13, 14) during the first conflict block in the revised goal neglect DCCS.

Figure 5. Temporal changes in the oxy-Hb (red) and deoxy- $\mathrm{Hb}$ (blue) concentration within the right lateral prefrontal areas (channel 4) during the redundant block of each cycle in the revised goal neglect DCCS. 
Figure 1

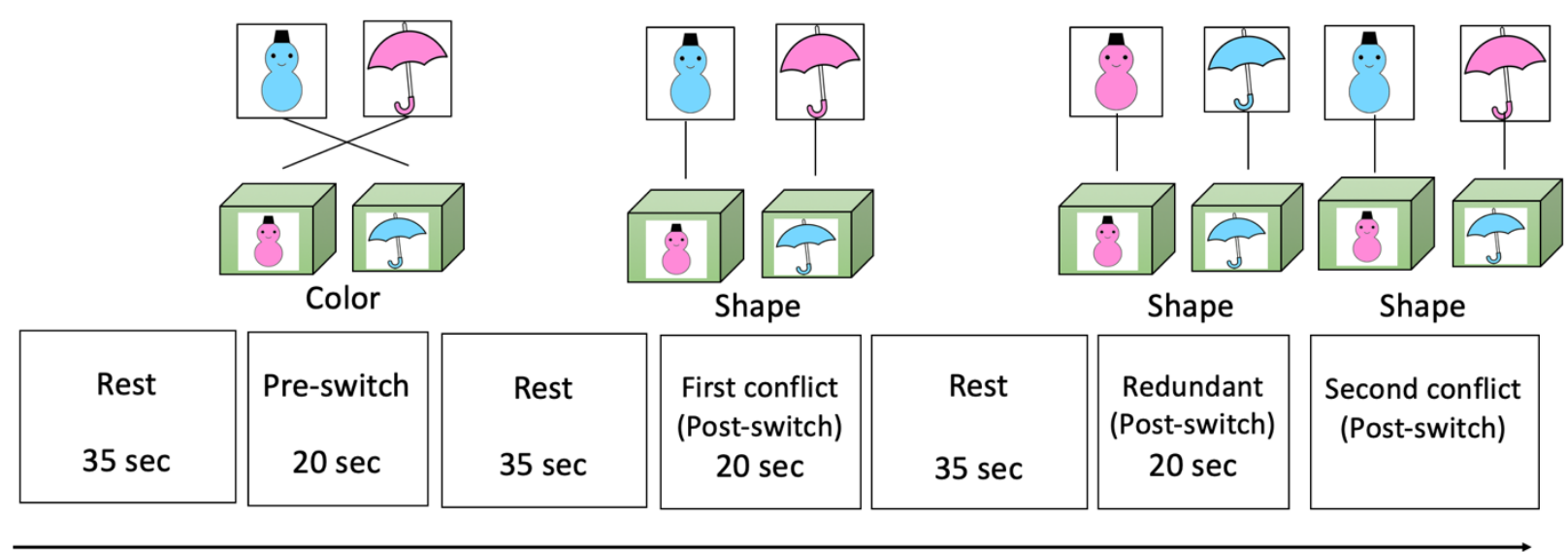


Figure 2

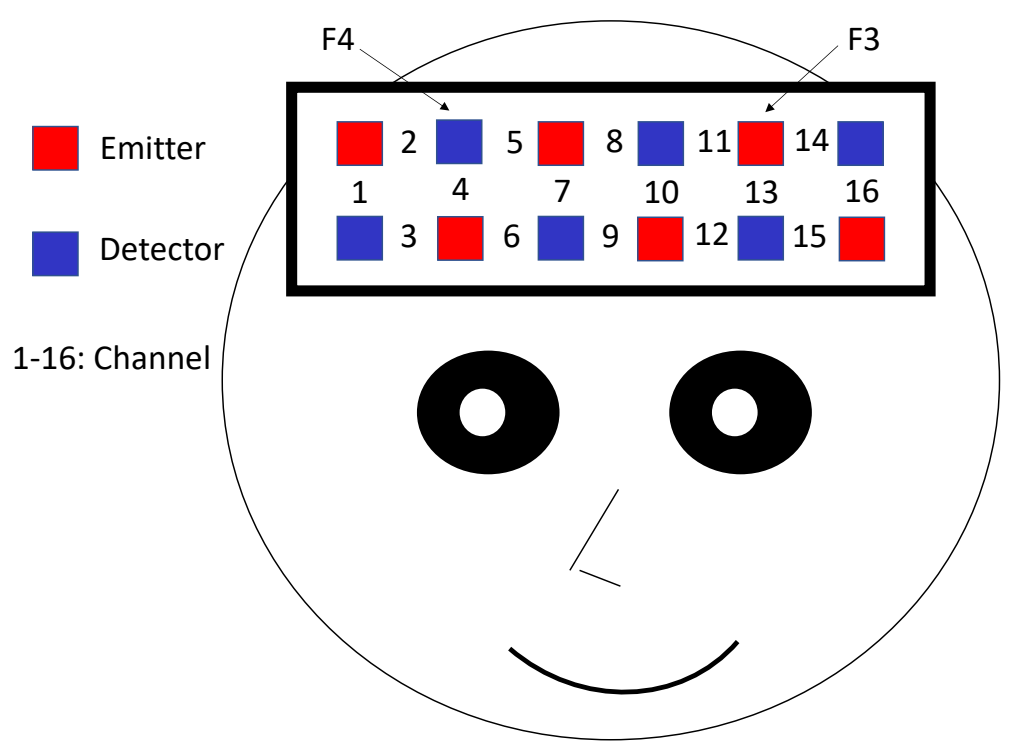


Figure 3

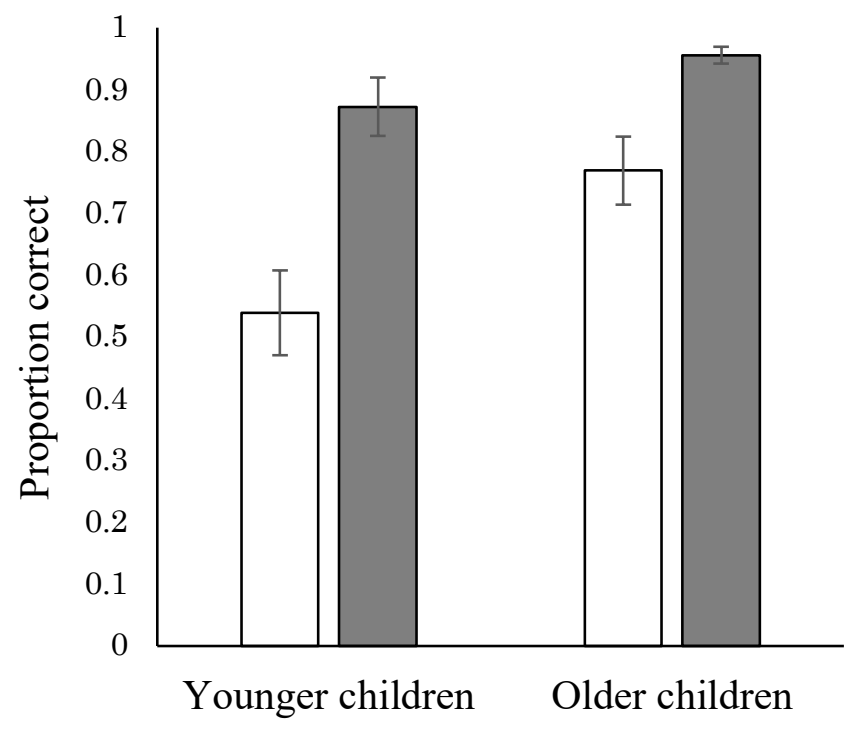

$\square$ Redundant deck

$\square$ Conflict deck 
Figure 4
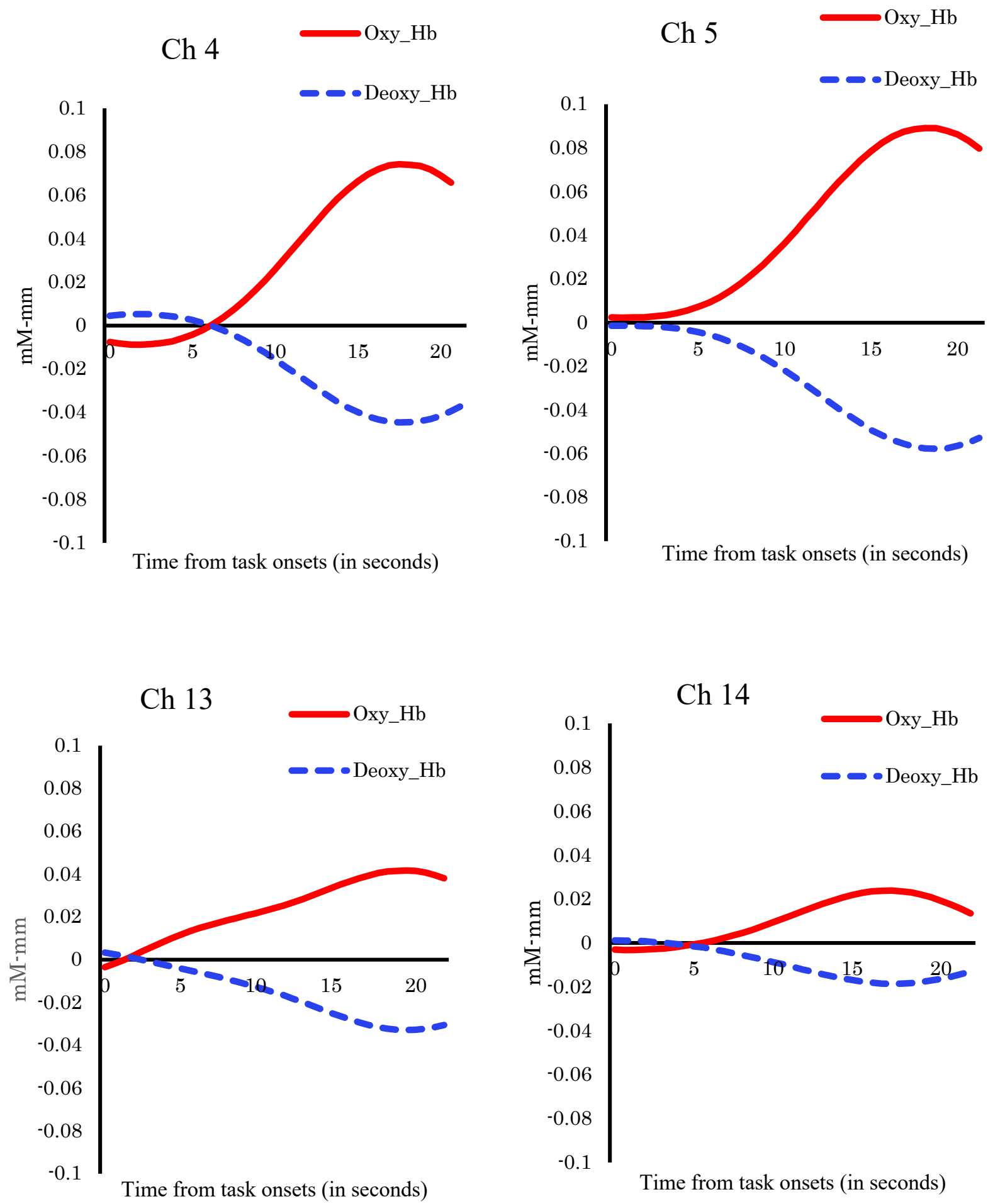
Figure 5
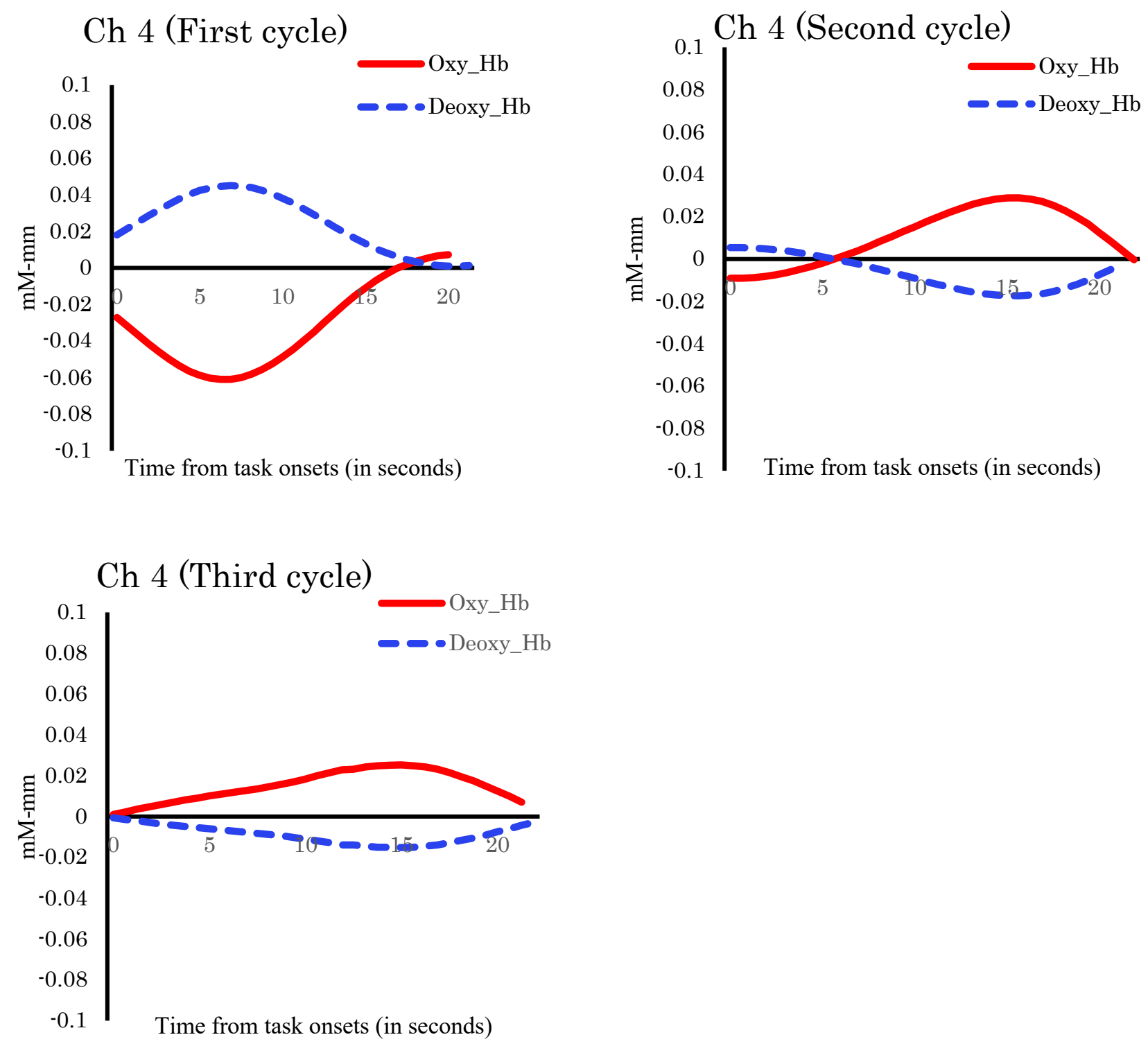